Bei Regenwetter werden große Mengen von Abwasser in kurzer Zeit ungereinigt in unsere Gewässer geleitet. Speicherbecken, die diese Einleitungen vermindern sollen, sind nicht nur teuer, sondern es ist auch ihr ökologischer Nutzen fragwürdig. Wie läßt sich der Zielkonflikt "Wasser bei Regen abführen - Schmutzstoffe zurückhalten" besser bewältigen? Welche Zusammenhänge zwischen der Abwassertechnik einerseits und der Ökologie anderseits sind heute für die Regenwettersituation bekannt? Welche Gewässerschutzstrategien lassen sich für die Zukunft daraus herleiten? Sind wir gewillt, auch die (hohen) Kosten dafür zu tragen?

\title{
Gewässerschutz bei Regenwetter
}

\author{
Vladimir Krejci, Jörg Lange und Wolfgang Schilling*
}

\begin{abstract}
The typical Central European urban drainage and receiving water system is described both from a historical and a technical perspective. Most common is the combined sewer system in which sanitary sewage and storm sewage are conveyed to the treatment plant in a single pipe.

During storms this system is overloaded and large quantities of sewage by-pass the treatment plant as so-called combined sewer overflows (CSO) without further treatment.

Sewage generating and transport processes in urban drainage systems are relatively well known. They can be simulated today with fair accuracy to predict measures against adverse effects in urban areas.

Today, simulation models are an indispensable tool to describe the characteristics of CSO.

Knowledge on the ecological effects of CSO is much more limited, though. There is some evidence, however, that adverse impacts to receiving water biota are primarily resulting from morphological modifications of receiving water courses rather than resulting from CSO.

A future urban drainage strategy should therefore be linked to the revitalization of receiving water courses, and focus on the deceleration of storm runoff by infiltration, the separation of strong and weak sewage at the source, and the allocation of measures in a site-specific and problem-oriented manner. Storage ponds to reduce combined sewer overflows should only be applied if specific receiving water requirements can be identified.
\end{abstract}

Keywords: combined sewer overflows, pollution control, rainfall, receiving waters, sewer systems, storage ponds, urban drainage

* Postadresse : Dr. W. Schilling Abteilung Ingenieurwissenschaften Eidgenössische Anstalt für Wasserversorgung, Abwasserreinigung und Gewässerschutz (EAWAG)

CH-8600 Dibendorf (Schweiz)

\section{Wie hat sich die Siedlungsentwässerung entwickelt?}

Die ältesten Überreste von Einrichtungen zur Siedlungsentwässerung stammen aus der Zeit vor fünf Jahrtausenden. Geradezu modern erscheinen die Einrichtungen der sogenannten Indus-Zivilisation (im heutigen Pakistan und Indien) vor etwa 4500-3500 Jahren: Es gab schon Klosetts, Badezimmer und StraBenkanalisationen.

Spätestens seit dem Bau der "Cloaca maxima" in Rom ist die Siedlungsentwässerung im Mischsystem bekannt. Die Cloaca maxima ist ein noch heute erhaltener riesiger, unterirdischer Kanal, der einem ursprünglich natürlichen Gewässerlauf folgt und nach dem Jahre 200 v.Chr. ein Gewölbe erhielt. Zusammen mit weiteren sechs Sammelkanälen führte die Cloaca maxima also bereits vor mehr als 2000 Jahren das Schmutzund Regenwasser der Stadt Rom ab. Plinius der Ältere (23-79) schreibt über diese Kanäle in seiner Naturalis Historia: "Es stoßen durch unterirdische Gänge sieben Bäche zusammen, welche auf ihrem schnellen, reißenden Lauf alles, was ihnen im Weg liegt, mit sich nehmen und wegschwemmen, obendrein durch Regengüsse verstärkt Grund und Boden erschüttern, die zuweilen rückwärts eindringenden Fluten des Tibers aufnehmen, gegen dieselben von der anderen Seite herankämpfen; und dennoch steht das Ganze unerschütterlich fest. Immerwährend werden darin so große Lasten fortgeschleppt, ohne daß die Gewölbe nachgeben" [1].
Schon im 17. Jahrhundert wurde die Einleitung von ungereinigtem "Abtrittwasser" in Paris verboten, da man eine schädliche Wirkung auf natürliche $\mathrm{Ge}$ wässer erkannt hatte. $\mathrm{Zu}$ jener Zeit war es üblich, Fäkalien entweder in Gruben oder in Tonnen zu sammeln und sie dann in regelmäßigen Abständen für die Düngung der Felder zu benutzen.

Die bakterielle Verseuchung des Trinkwassers durch Abwasserversickerung im Boden wurde erst im Jahre 1883 erkannt. Vorher glaubte man, daß anstekkende Krankheiten vor allem durch vom Boden und Grundwasser aufsteigende giftige Gase verursacht werden. Erst die Entdeckung der Cholerabakterien durch Robert Koch und das Verständnis ihrer Ausbreitung (vom Stuhlgang der Cholerakranken über den Boden zum Trinkwasser) machte die ungeheure Bedeutung einer peinlichen Trennung von Fäkalien und Trinkwasser bewußt.

Das relative große Wasserdargebot in Mitteleuropa ermöglichte die Einführung von zentralen Wasserversorgungen, Bädern und Spültoiletten. Damit nahm die Menge des Schmutzwassers derart zu, daß das Tonnen- oder Grubensystem aufgegeben werden mußte (in Zürich erst um $1920^{[1]}$ ) und das Schmutzwasser in die Gewässer abgeleitet wurde. Um Kellerräume trocken zu halten, begann man, auch das Sicker- und Drainagewasser abzuleiten (Figur 1). Komfortsteigerungen in den Haushalten brachten immer mehr Schmutzwasser mit sich, dessen Einleitung die Verschmutzung vor allem kleinerer Fließgewässer so erhöhte, daß sie nicht mehr für andere Zwecke genutzt werden konnten (Trinkwasser, Fischerei). Man begann, sie zu verdolen (das heißt zu verrohren). In der Stadt Zürich sind von urspünglich $160 \mathrm{~km}$ offener Bachläufe bis zum Jahre 1986 nur noch $60 \mathrm{~km}$ übriggeblieben. Gegen Ende des vorigen Jahrhunderts entstanden zunächst in den größeren Städten Mitteleuropas Entwässerungssysteme nach dem Prinzip der "Schwemmkanalisation", also der Nutzung des (Regen-)Wassers als Transportmittel für Fäkalien und andere unerwünschte Stoffe.

In der Schweiz und in Deutschland ist die Schwemmkanalisation zum zentralen Prinzip der Siedlungsentwässerung geworden.

Als das Selbstreinigungsvermögen auch größerer Fließgewässer infolge der zunehmenden Abwassereinleitungen er- 
Tabelle 1. Entwicklung der Abwassertechnik [1-3],

\begin{tabular}{ll}
\hline $5000-3000$ v.Chr. & Rohre und offene Halbschalen aus gebranntem Ton zur Siedlungs- \\
& entwässerung im Euphrat-Tal \\
2500 v.Chr. & Abwasserleitungen in Mesopotamien \\
$2500-1500$ v.Chr. & Badezimmer, Klosetts und Straßenkanalisation der Indus-Zivilisation \\
2000 v.Chr. & Rohre für Wasserversorgung, Regenwasserspeicher und Abwasseranlagen \\
& im Palast von Knossos \\
1700 v.Chr. & Hammurabi, Herrscher im Zweistromland, erläßt ein Gesetz zu Bewässe- \\
& rung und Hochwasserschutz \\
$624-546$ v.Chr. & Thales von Milet: Idee vom Kreislauf des Wassers \\
300 v.Chr. & Ausbau der Kanalisation in Rom \\
1591 & Vorschläge zur Abwasserklärung in London \\
1660 & Wasserklosetts (WC) in England und Frankreich \\
ab 1760 & Rieselfelder für Abwasser \\
1830 & Schwere Cholera-Epedemie in London \\
$1840-1850$ & Bau der Kanalisation in London \\
1848 & Erste moderne Kanalisation Deutschlands in Hamburg \\
1873 & Kanalisation in Berlin \\
1877 & Erster Verein zur Reinerhaltung von Wasser, Boden und Luft, \\
& gegründet in Köln \\
1884 & Typhus-Epidemie in Zürich \\
1888 & Fischereigesetz in der Schweiz (mit Vorgaben zum Gewässerschutz) \\
1892 & Biologische Abwasser-Reinigungsverfahren in England; \\
1895 & Cholera-Epidemie in Hamburg \\
vor 1908 & Erstes Klärbecken in Deutschland \\
1916 & Erste biologische Untersuchungen der Gewässerverunreinigung durch \\
1971 & Abwasser \\
1975 & Erste mechanisch-biologische Kläranlage in der Schweiz (St. Gallen) \\
1976 & Gewässerschutzgesetz in der Schweiz \\
1977 & Verordnung über Abwassereinleitungen in der Schweiz \\
1991 & Abwasserabgabengesetz in Deutschland \\
& ATV-Bemessungsvorschrift A 128 für Mischwasserbecken \\
& Rund 9000 Mischwasserbecken in Deutschland (alte Bundesländer) \\
&
\end{tabular}
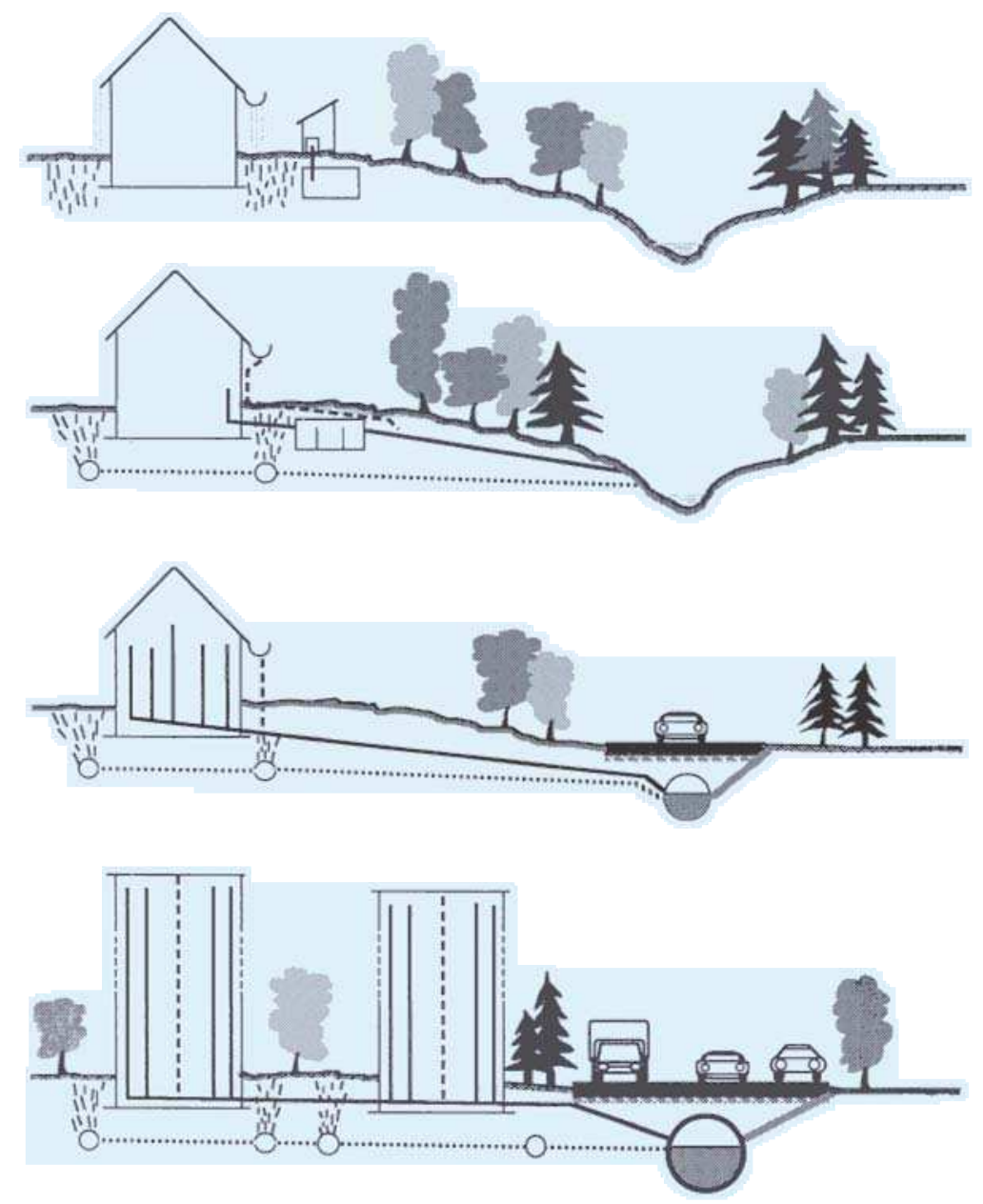

schöpft war, begann man um die Jahrhundertwende mit dem Bau der ersten Kläranlagen (vergleiche Tabelle 1). In Deutschland (alte Bundesländer) und in der Schweiz sind heute um $90 \%$ der Einwohner an Kläranlagen angeschlossen, so daß erhebliche Einleitungen von ungereinigtem Abwasser in die Gewässer nur noch bei Regenwetter vorkommen. Die Problematik dieser "Mischwasserentlastungen" wird im folgenden diskutiert. Das Wachstum der Städte erforderte laufende Kapazitätsanpassungen der Kanalisationen und in zunehmendem Maße auch eine konstruktive Sanierung der zum Teil über 100 Jahre alten gemauerten Sammler.

\section{Wohin fließt unser Abwasser heute?}

Kaum eine Angelegenheit ist alltäglicher geworden als die Beseitigung von Fäkalien und anderen Abfallstoffen mittels Wasserspülung. Doch was nach dem Spülen mit unserem Abwasser passiert, entzieht sich in aller Regel der allgemeinen Aufmerksamkeit. Das ist nicht weiter verwunderlich, verschwindet doch das Abwasser in unterirdischen Kanalisationsleitungen, um erst an einem ganz anderen Ort wieder hervorzukommen. Diese Leitungen sind allein in Deutschland, öffentliche und private aneinandergereiht, länger als die Entfernung Erde-Mond. Aber ungeachtet der immensen Aufwendungen für die Abwasserentsorgung (vergleiche Tabelle 2) und ihrer Bedeutung für unsere Umwelt interessiert sich die Öffentlichkeit nur wenig für sie. Über eine aktuelle Ausnahme berichtet die Neue Zürcher Zeitung vom 29. Januar 1992: »Wieviel kostet Regenwasser? Im Dezember 1991 haben die Abwassergebührenrechnungen der Wasserentsorgung zahlreiche Liegenschafteneigentümer in der Stadt Zürich verunsichert und verärgert. Die verlangten Gebühren waren massiv er-

Figur 1. Historische Entwicklung der Siedlungsentwässerung. Mit der Einführung zentraler Wasserversorgungen, von Bädern und Spültoiletten sammelte sich bald soviel Schmutzwasser an, daß das Tonnen- oder Grubensystem (a) aufgegeben werden mußte und das Schmutzwasser in die Gewässer abgeleitet wurde. Um Keller besser nultzen zu können, wurde auch das Sickerund Drainagewasser abgeleitet (b). Die Schmutzwassermengen stiegen mit dem Lebensstandard. Aus manchen Bächen wurde eine "Schwemmkanalisation" (c). Das Wachstum der Städte erforderte bis heute Kapazitätsanpassungen (d). 
Tabelle 2. Siedlungsentwässerung in Zahlen (Stand um 1990 [4, 5], teilweise geschätzt)

\begin{tabular}{lll}
\hline & $\begin{array}{l}\text { Schweiz } \\
\text { pro Einwohner }\end{array}$ & gesamt \\
\hline Länge öffentlicher Kanäle & $4-6 \mathrm{~m}$ & $30000 \mathrm{~km}$ \\
Volumen vorhandener Mischwasserspeicher & $0.1 \mathrm{~m}^{3}$ & $0.5 \mathrm{Mio} \mathrm{\textrm {m } ^ { 3 }}$ \\
Kosten vorhandener Mischwasserspeicher & $>200 \mathrm{sFr}$ & $\approx 1.5 \mathrm{Mrd} \mathrm{sFr}$ \\
Zusätzlicher Bedarf Mischwasserspeicher & $0.1 \mathrm{~m}^{3}$ & $0.5 \mathrm{Mio} \mathrm{\textrm {m } ^ { 3 }}$ \\
Kosten zusätzlicher Mischwasserspeicher & $200-400 \mathrm{sFr}$ & $>1.5 \mathrm{Mrd} \mathrm{sFr}$ \\
Sanierung öffentlicher Kanalisation & $>5000 \mathrm{sFr}$ & $>30 \mathrm{Mrd} \mathrm{sFr}$
\end{tabular}

höht, denn inbegriffen war plötzlich auch eine Gebühr für das Regenwasser, die "Meteorwasserkomponente"... Die Entsorgung von Regenwasser, das über dasselbe Kanalsystem entsorgt wird wie das Schmutzwasser, verursacht nämlich 28 Prozent der Gesamtkosten der Wasserentsorgung..."

In den meisten Siedlungen Mitteleuropas wird heute häusliches Schmutzwasser bei Trockenwetter zu einer Kläranlage geleitet und gelangt von dort mehr oder weniger gut gereinigt in die sogenannten Vorfluter (Gewässer wie Bäche, Flüsse und Seen). Neben diesem einen Teil der Abwässer entsteht in Siedlungsgebieten ein anderer Teil, wenn Regenwasser von versiegelten Flächen wie Straßen und Dächern abfließt ("Meteorwasser").

Als Abwässer werden im weiteren Sinne sämtliche Wässer bezeichnet, die aus überbauten Gebieten abgeleitet werden müssen, aus Haushalten, Gewerbe und Industrie, einschließlich Kühlwasser, Schneeschmelz- und Sickerwasser. Dabei ist es gleichgültig, ob sie verschmutzt oder unverschmutzt sind ${ }^{[6]}$. Werden diese Abwässer gemeinsam in einem Rohr abgeleitet, so spricht man von Mischwasser.

Für diese Abwässer sind in Mitteleuropa im wesentlichen zwei Arten der Entwässerung üblich. Zum einen werden Regenwasser und Schmutzwasser in getrennten Kanälen abgeführt (Trennsystem, Trennkanalisation). Die Trennkanalisation ist vor allem in den Außenbezirken von Siedlungen anzutreffen. In Deutschland ist dieses Entwässerungsprinzip in den küstennahen Bundesländern stärker verbreitet, da das Regenwasser bei geringem Gefälle auf kurzem Wege oberflächlich in die Vor-

Figur 2. Typisches Abwassersystem in Mitteleuropa: das Mischsystem. Das gesamte Abwasser aus den Siedlungen (Dachabwässer, häusliche Abwässer, Straßenabwässer und Industrieabwässer) fließt im selben Kanal zur Kläranlage. Bei einem Starkregen fließt für kurze Zeit bis zum Hundertfachen des Trockenwetterabflusses (100 QTW) an der Kläranlage vorbei in die Gewässer. fluter geleitet werden kann, ohne es pumpen zu müssen. In der Regel wird im Trennsystem das Regenwasser weder zwischengespeichert noch gereinigt; Ausnahmen sind Regenwasserableitungen von besonders mit Schmutzstoffen belasteten Oberflächen wie Autobahnen, Flugplätzen oder Industrieanlagen. Die Trennkanalisation hat einige Nachteile: Auch verschmutztes Regenwasser gelangt unkontrolliert und ungereinigt in die Gewässer. Fehlanschlüsse von Schmutzwasserleitungen an Regenwasserkanäle verursachen ständige Gewässerverunreinigungen (bei alten Trennsystemen ist mit $5 \%$ und mehr falschen Anschlüssen zu rechnen).

Zum anderen werden Regenwasser und Schmutzwasser im selben Kanal abge-
Deutschland (alte Bundesländer)

\begin{tabular}{ll} 
pro Einwohner & gesamt \\
\hline $5 \mathrm{~m}$ & $285000 \mathrm{~km}$ \\
$>0.072 \mathrm{~m}^{3}$ & $>4.5 \mathrm{Mio} \mathrm{m}^{3}$ \\
$110 \mathrm{DM}$ & $7 \mathrm{Mrd} \mathrm{DM}$ \\
$\approx 0.24 \mathrm{~m}^{3}$ & $\approx 15 \mathrm{Mio} \mathrm{m}^{3}$ \\
$320-370 \mathrm{DM}$ & $20-23 \mathrm{Mrd} \mathrm{DM}$ \\
$>2250 \mathrm{DM}$ & $>140 \mathrm{Mrd} \mathrm{DM}^{2}$
\end{tabular}

führt (Mischsystem, Mischkanalisation; siehe Figur 2). Das Mischsystem kommt billiger zu stehen als das Trennsystem (einfaches statt doppeltes Rohrnetz) und ist das viel häufiger installierte. So sind in der Schweiz etwa $70 \%$, in den Niederlanden sogar $90 \%$ der Kanalnetze Mischkanalisationen. Die Menge Wasser, die bei starkem Regen durch ein Mischwassernetz abfließt, ist wesentlich gröBer als der Schmutzwasserabfluß bei Trockenwetter. Aus ökonomischen, aber auch aus verfahrenstechnischen Gründen werden Kläranlagen in der Regel für das Doppelte der bei Trockenwetter fließenden Wassermenge dimensioniert. Bei ergiebigeren Regen wird diese Menge jedoch schnell übertroffen. Das überschüssige Wasser muß zur Entlastung

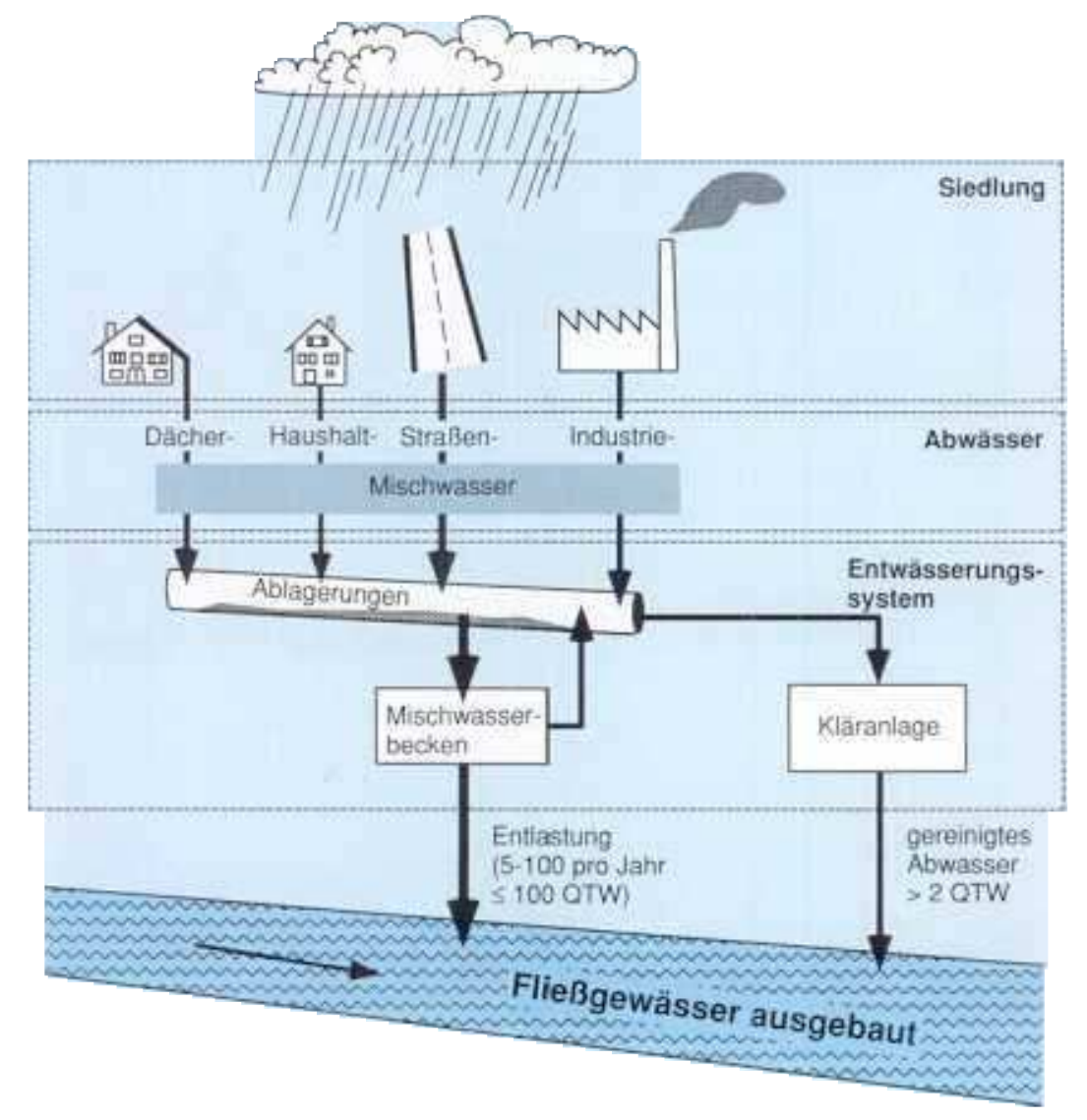


durch Mischwasserïberläufe (auch Regenüberläufe genannt) ungeklärt in die Gewässer abfließen (Figur 3).

Bereits in den sechziger und siebziger Jahren haben zahlreiche Messungen gezeigt, daß Mischwasser stark variierende, oft aber beachtliche Schmutzfrachten enthält. Diese Untersuchungen haben zu einem besseren Verständnis der Transportprozesse in Entwässerungssystemen und daher zu Änderungen der Gewässerschutz-Strategie geführt. Danach wurden vor allem in Deutschland und in der Schweiz Mischwasserspeicher gebaut, welche die Schmutzfrachten zurückhalten sollten ("end-ofpipe strategy"). Bevor das Mischwasser in die Gewässer fließt, wird es in unterirdischen Becken gespeichert und nach Regenende zur Kläranlage weitergeleitet (Figur 4). Erst wenn auch diese Speicher gefüllt sind, fließt ungereinigtes Mischwasser in die Gewässer. Die Kapazität der Mischwasserbecken in unseren Siedlungen ist aber bei kräfigem Regen bald erschöpft.

Um die infolge der Urbanisierung immer mehr anschwellenden Abflüsse bewältigen zu können, wurden die noch offenen Gewässerläufe kanalisiert und mit hydraulisch effizienten Querprofilen versehen. Ein betonierter Kanal mit rechteckigem Querschnitt (Figur 5) bringt für eine vorgegebene Grundfläche bei weitem die höchste Abflußleistung! Erst in jüngster Zeit strebt man an, diese Entwicklung nach der Devise "GewässerRenaturierung" umzukehren.

Im Laufe dieses Jahrhunderts hat der Mensch ständig mehr Abwasser mit ständig wachsenden Schmutzfrachten in natürliche Gewässer abgeleitet. Dadurch sind viele Bäche zu Abwasserkanälen degeneriert.

Ein Blick über die Grenzen der Schweiz und Deutschlands hinaus zeigt, daß Gewässerschutz bei Regenwetter in allen Industrieländern ein ungelöstes Problem ist, dem man mit den unterschiedlichsten Maßnahmen beizukommen sucht. In den Niederlanden wird versucht, das Problem durch zusätzlichen Speicherraum in Form großkalibrierter Kanalnetze zu lösen. In den USA gibt es Mischkanalisationen fast nur in den alten Städten (für etwa $25 \%$ der Bevölkerung), der überwiegende Teil der Einwohner wird im Trennsystem entsorgt. Spezielle Maßnahmen zur Verminderung der Mischwassereinleitungen sind bisher rar. England verlagert die Verschmutzungs-

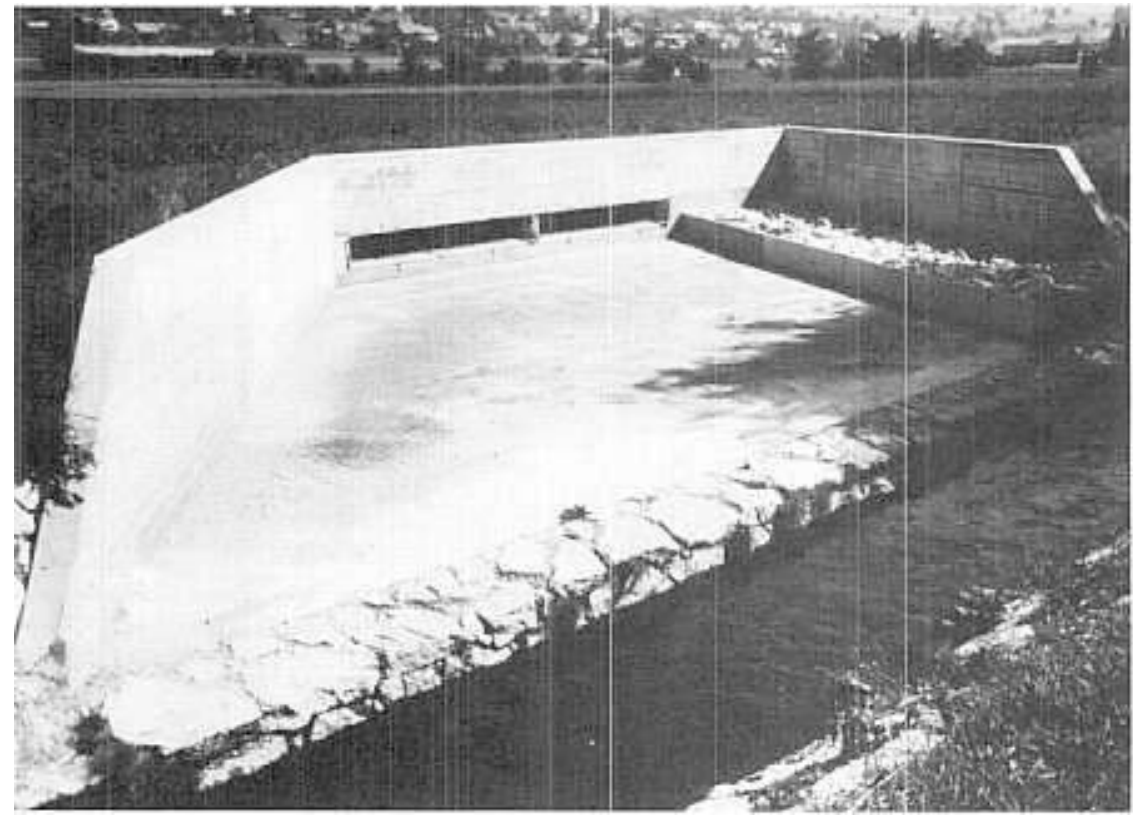

Figur 3. Mischwasserüberlauf in ein kleines Fließgewässer. Große Mischwassermengen an dieser Stelle erfordern große Bauwerksabmessungen. Schon bei kurzem Starkregen sind die eingeleiteten Abwassermengen hier wesentlich größer als der natürliche Durchfluß im Gewässer. Die hohe Fließgeschwindigkeit des eingeleiteten Abwassers erfordert eine massive Befestigung des Bachbetts.

problematik, begünstigt durch seine Insellage, häufig ins Meer. Abwasser wird hier oft über "deep sea outfalls" so weit entfernt von der Küste ins Meer geleitet, daß in Küstennähe keine hohen Schadstoffkonzentrationen mehr auftreten. In Ost- und Südeuropa kommen die Sorgen bei Trockenwetter noch vor denen bei Regenwetter, das heißt, vielenorts fehlen überhaupt funktionierende Kläranlagen. Einzig in Skandinavien wird ein Bündel differenzierter Maßnahmen ausprobiert, wobei jedoch

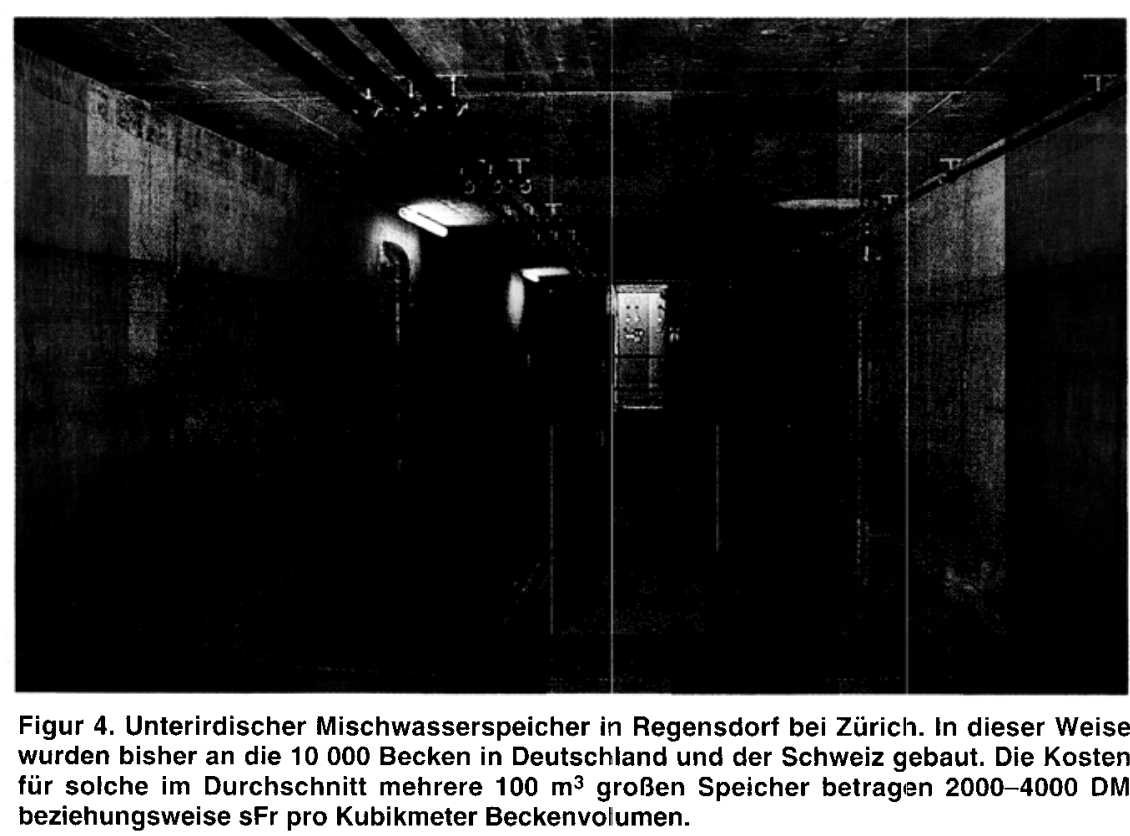

Figur 4. Unterirdischer Mischwasserspeicher in Regensdorf bei Zürich. In dieser Weise wurden bisher an die 10000 Becken in Deutschland und der Schweiz gebaut. Die Kosten für solche im Durchschnitt mehrere $100 \mathrm{~m}^{3}$ großen Speicher betragen 2000-4000 DM beziehungsweise sFr pro Kubikmeter Beckenvollumen.

die Mischsysteme keine große Rolle spielen ${ }^{[7]}$.

In den meisten Entwicklungsländern ist die Situation gänzlich anders: Dort gibt es fast überall weder Kanalisationen noch Kläranlagen. Große Bedeutung für die Zukunft dieser Länder hat die Frage, ob sich dort eine Entwicklung nach dem Vorbild der Industrieländer wiederholen muß oder ob es gelingen wird, neue Strategien zur Abwasservermeidung und Abwasserentsorgung einzuschlagen. 


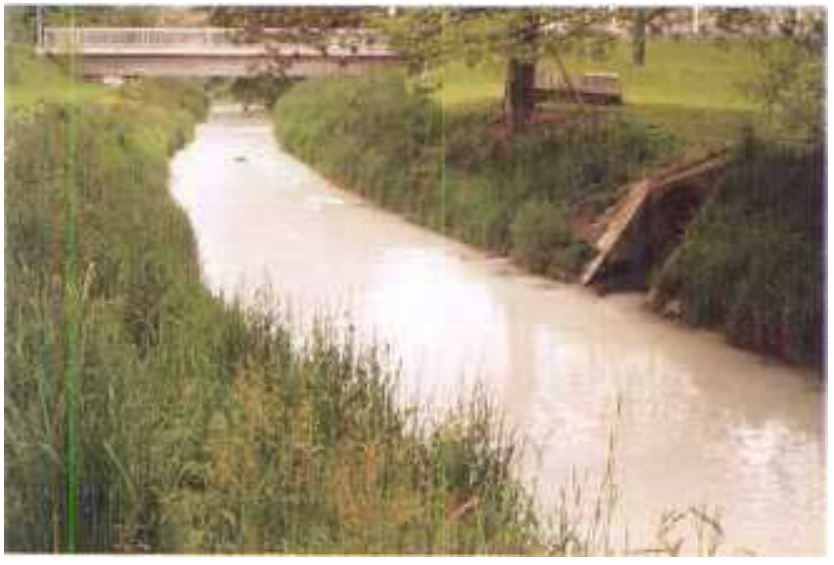

Figur 5. Chriesbach vor (links, im Jahre 1970) und nach (rechts, im Jahre 1987) dem Ausbau. Durch den Ausbau dieses Gewässers in Dübendorf bei Zürich wurde dessen Durchflußkapazität von $15 \mathrm{~m}^{3} / \mathrm{s}$ auf $90 \mathrm{~m}^{3} / \mathrm{s}$ erhöht, um zusätzlich die Regenwasserabflüsse aus

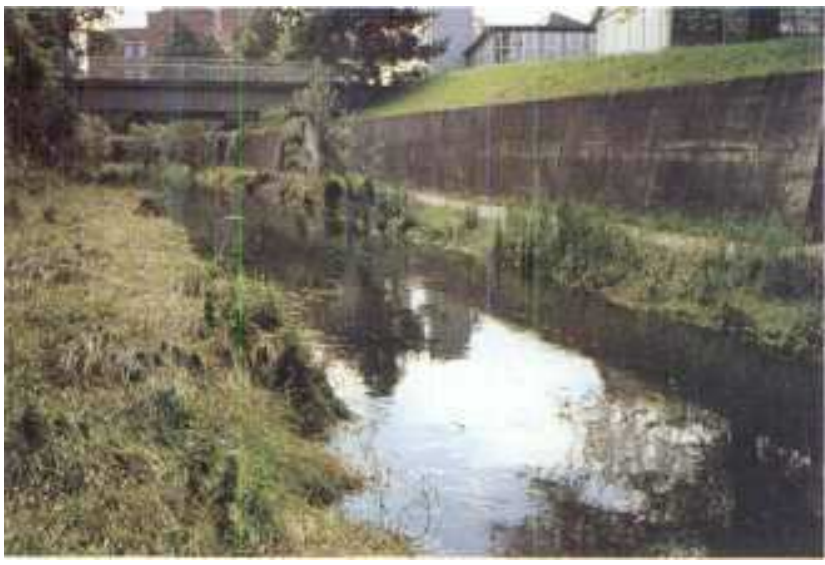

dem Siedlungsgebiet aufnehmen und abführen zu können. Die Konkurrenz zwischen geringem Flächenangebot und hydrologischen Erfordernissen der Wasserableitung führt zu derartigen Extremlösungen.

\section{Haben sich die bisher befolgten Entwässerungskonzepte bewährt?}

Die traditionelle Entwässerungsstrategie, in der Generationen von Fachingenieuren unterwiesen wurden, läßt sich durch ein Zitat aus dem Standardwerk Kanalisation von Hörler aus dem Jahre 1966 prägnant beschreiben ${ }^{\text {[8] }}$ : »Aufgabe der Ortsentwässerung ist es, sämtliche Abwässer so vollkommen und so schnell als möglich zu sammeln und aus dem Bereich menschlicher Siedlungen zu entfernen, ohne Belästigung der Bewohner, ohne Beeinträchtigung des Verkehrs und ohne Schädigung der oberund unterirdischen Gewässer.«

Bei der Befolgung dieses Grundsatzes wurden zwar die hygienischen und ästhetischen Bedürfnisse sowie die Forderungen nach Sicherheit und Komfort hinsichtlich der Regenwasserableitung befriedigt, aber der darin enthaltene Zielkonflikt wurde in der Praxis zu Ungunsten des Gewässerschutzes "gelöst".

\section{Der Zielkonflikt:}

Einerseits soll Regenwasser so schnell wie möglich abgeleitet werden,

um Rückstau und Überschwemmungen

in den Siedlungen zu vermeiden,

anderseits enthält das Mischwasser

Schmutzstoffe, die nicht in das Gewässer gelangen sollen.

Mit der Verordnung über Abwassereinleitungen ${ }^{[6]}$ der Schweiz wurde den Qualitätszielen für die Gewässer eine größere Bedeutung zuerkannt: Neben

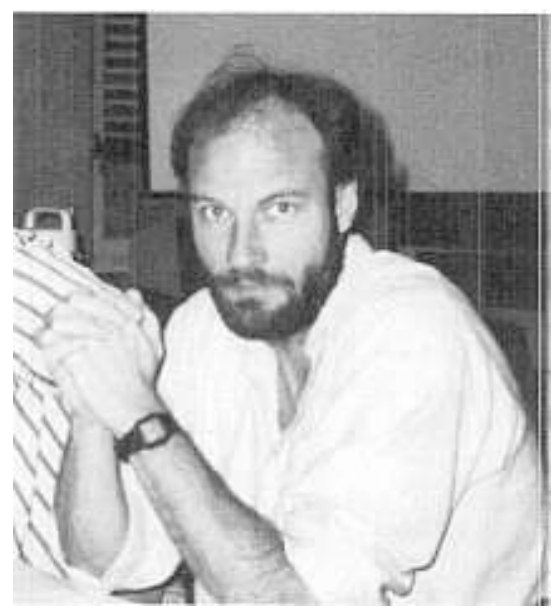

Wolfgang Schilling: Geboren 1954 in Hannover Niedersachsen. Studium des Bauingenieurwesens an der Universität Hannover; Diplom 1979; Aufbaustudium am Massachusetts Institute of Technology, Cambridge, USA; Promotion 1983 über Niederschlagvorhersagen; 1984 Gastwissenschaftler an der Colorado State University, Fort Collins, USA; Gastprofessur 1985 an der University of Ottawa, Canada; 1987 Habilitation für das Lehrgebiet "operationelle Wasserwirtschaft". Seit 1988 wissenschaftlicher Mitarbeiter der Abteilung für Ingenieurwissenschaften an der Eidgenössischen Anstalt für Wasserversorgung, Abwasserreinigung und Gewässerschutz (EAWAG) in Dübendorf bei Zürich; Lehraufträge an der ETH Zürich, Grundlagenforschung, Weiterbildung und Beratung für das Gebiet "Siedlungsentwässerung und Gewässerschutz".

den Grenzkonzentrationen der Abwassereinleitungen waren fortan auch die resultierenden Kennwerte im Gewässer bestimmend für den Gewässerschutz. Vor allem die im Anhang der Verordnung aufgeführten tolerierbaren StoffKonzentrationen bei Trockenwetter haben dem Praktiker brauchbare Hinweise für eine weitergehende Abwasserreinigung (Phosphor- und Stickstoffelimination, Filtration) gegeben. Die spezifischen Probleme der Gewässerverunreinigung während Regenwetter wurden jedoch in der Verordnung nicht explizit berücksichtigt. Auch fehlten - in Analogie zum Trockenwetter - konkrete Hinweise (Toleranzwerte) für die Planung von Maßnahmen bei Regenwetter.

Die Verordnung blieb also die Antwort auf die Frage nach der Priorität der weitergehenden Abwassereinigung vor der Regenwasserbehandlung schuldig. Diese Frage wurde in einer umfangreichen regionalen Fallstudie untersucht ${ }^{[9]}$. Dabei wurde deutlich, wie komplex allein die Identifikation der Gewässerschutzpro- bleme bei Regenwetter ist und wie schwierig ökologisch begründete Toleranzlimits für die Regenwettersituation festzulegen sind. Insbesondere war es anhand der dokumentierten Regenereignisse nicht gelungen, einen ökologischen Schaden als Folge von Mischwassereinleitungen zu identifizieren. Es stellte sich heraus, daß die meisten Mischwasserbecken weder bei der Planung noch im Betrieb auf die spezifischen Verhältnisse im Einzugsgebiet und vor allem im Gewässer abgestimmt worden waren.

\section{Die bisher übliche}

Gewässerschutz-Strategie hat sich nur partiell bewährt:

Probleme wurden von den Siedlungen zum Teil ins Gewässer verlagert (Beispiel: Abflußmengen) und als "Reinhaltung des Wassers im Gewässer" (im Gegensatz zu

"Erhaltung des Lebensraums Gewässer") zu eng definiert. 
Diese Erkenntnisse führten zu folgenden Vorschlägen ${ }^{[10]}$ :

1. Bevor Maßnahmen zur Mischwasserbehandlung ergriffen werden, sollten Probleme im Entwässerungssystem und im Gewässer identifiziert werden.

2. Die Maßnahmen sollen problemorientiert und den lokalen Gegebenheiten angepaßt sein.

3. Maßnahmen an der Quelle (das heißt zur Abwasser- und Schmutzstoffvermeidung) sollen Priorität vor "end-ofpipe"-Maßnahmen haben.

4. Für die existierenden Entwässerungssysteme sollte sichergestellt werden, daß Mischwasserentlastungen erst dann vorkommen, wenn tatsächlich sämtliche Kapazitätsreserven ausgeschöpft sind (Speicher-, Transport- und Behandlungskapazität).

Diese Empfehlungen konfrontierten die Praktiker plötzlich mit Problemen, die sie aus ihrer bisherigen Tätigkeit nicht kannten. Für solche Aufgaben waren sie nicht nur unvorbereitet; sie wurden auch seitens der Gewässerökologen nicht entsprechend unterstützt und haben deswegen die vorgeschlagene Strategie nicht angewendet.

Die für den Gewässerschutz zuständigen Aufsichtsbehörden in der Schweiz akzeptierten jedoch einen Teil der Kritik. Sie unterstützten in der Folge einige Untersuchungen über die Effizienz von Mischwasserbecken, die Entwicklung von Alternativen (zum Beispiel Siebanlagen) sowie von Maßnahmen an der Quelle (Fremdwasser-Reduktion, Versickerung von unverschmutztem Abwasser). Gleichzeitig bemühte sich die Forschung, Wirkungszusammenhänge zwischen Mischwassereinleitungen und ökologischen Beeinträchtigungen aufzudecken.

Auch wurde die bisherige "Philosophie" der Siedlungsentwässerung (schnelle Ableitung sämtlicher Abwässer) diskutiert und in Frage gestellt. Der Verband Schweizerischer Abwasserfachleute (VSA) revidierte seine Richtlinien ${ }^{[11]}$ unter der Prämisse eines ausgewogenen Verhältnisses zwischen Entwässerungskomfort und Gewässerschutz und unterstützt die Anwendung "maßgeschneiderter" Vorkehrungen.

In Deutschland wurde ebenfalls der Beeinflussung der Fließgewässer durch Mischwassereinleitungen in der letzten Zeit wesentlich mehr Aufmerksamkeit gewidmet. Vorläufig gelten die emissionsorientierten generellen Anforderungen an die Einleitungen ${ }^{[12]}$. Diese Mindest- oder Normalanforderungen werden momentan in Zusammenarbeit von Naturwissenschaftlern und Inge-

nieuren durch weitergehende Anforderungen ergänzt, die sich am Gewässer orientieren und lokalspezifisch wirksam werden sollen ${ }^{[13]}$.

\section{Zweifel an der traditionellen Gewässerschutz-Strategie werden zunehmend akzeptiert. Eine Trendwende zeichnet sich $a b$, zeitigt aber noch kaum praktische} Resultate.

\section{Wie lassen sich Mischwassereinleitungen charakterisieren?}

Aufgrund von Beobachtungen und Messungen sind die Abfluß- und Stofftransportprozesse in Siedlungsgebieten bei Regenwetter heute prinzipiell bekannt. Während etwa 400 Stunden der rund 1000 Stunden Regenwetter im Jahr regnet es so stark ( $a b$ ca. $1 \mathrm{~mm} / \mathrm{h}$ entsprechend $1 \mathrm{Liter} / \mathrm{h} \cdot \mathrm{m}^{2}$ ), daß die entstehenden Mischwassermengen den doppelten Trockenwetterabfluß und damit die Kapazität der Kläranlage überschreiten. Das überschüssige Abwasser wird kann dann bis zu 100mal so groß sein wie der Abfluß bei Trockenwetter. Es kann zu Rückstau im Kanalnetz und manchmal sogar zu Überschwemmungen kommen.

Im ersten Schritt einer Weiterentwicklung der Maßnahmen zum Gewässerschutz bei Regenwetter müssen die Belastung des Gewässers durch Abwasser und dessen Inhaltsstoffe charakterisiert werden. Grundsätzlich sind die Abwassermengen und die Konzentrationen der darin enthaltenen Stoffe meßbar. Der Aufwand dafür ist jedoch sehr groß: Sämtliche Überläufe eines Kanalnetzes (in größeren Städten Dutzende) müssen mit kontinuierlich aktivierten Meßgeräten ausgestattet werden, die bei Regenbeginn in schneller Folge den Durchfluß registrieren und Proben für die Analyse der Inhaltsstoffe nehmen.

Viel einfacher können mit numerischen Simulationsmodellen die aus der gemessenen Regenintensität resultierenden Abwassermengen und die darin enthaltenen Stoffkonzentrationen berechnet werden. Solche Modelle wurden in den letzten zwei Jahrzehnten aus international verfügbaren Meßergebnissen erstellt und sind heute für die Ingenieurforschung und -praxis ein unentbehrliches Werkzeug.

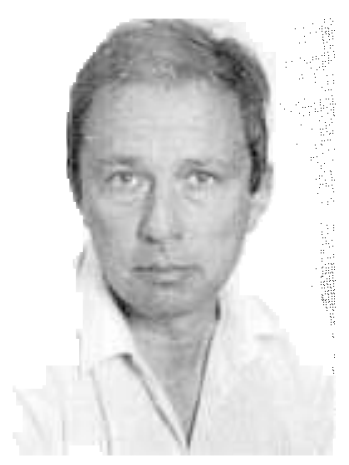

Vladimir Krejci: Geboren 1939 in Prag, Tschechoslowakei. Studium des Wasserbaus und der Kulturtechnik an der Technischen Hochschule Prag; Diplome 1962 und 1965; Nachdiplomstudium der Umweltwissenschaften an der EPF Lausanne. Seit 1969 wissenschaftlicher Mitarbeiter der Abteilung für Ingenieurwissenschaften an der Eidgenössischen Anstalt für Wasserversorgung, $A b$ wasserreinigung und Gewässerschutz (EAWAG) in Dübendorf bei Zürich; Leiter der EAWAG-Projekte "Glattstudie" 1976-1979 und "Gewässerschutz im ländlichen Raum" 1980-1982; 1984-1985 Gastwissenschaftler an der University of California, Davis, USA; Mitglied technischer Kommissionen in VSA und ATV; unterrichtet seit 1981 Abwassertechnik an der Ingenieurschule Zürich.

dann entweder in die Vorfluter abgeleitet oder in Becken zwischengespeichert.

Während 100 bis 200 Stunden oder 20 bis $30 \mathrm{mal}$ pro Jahr ist die Regenmenge so groß, daß vorhandene Mischwasserspeicher überlaufen. Sie haben etwa ein Volumen, das $2 \mathrm{~mm}$ Regen entspricht. Um jeden Überlauf zu verhindern, wären Speicher erforderlich, die mindestens zehnmal größer als die heute üblichen sind.

Wesentlich seltener (im Durchschnitt nur wenige Minuten pro Jahr) ist die Regenintensität so stark, daß die Kanalrohre vollständig gefüllt sind und das Regenwasser nicht mehr zur Gänze aufnehmen und ableiten können. Der Abfluß
Mit Simulationsmodellen ist es nun möglich, die Auswirkungen verschiedener technischer Maßnahmen im Entwässerungssystem für unterschiedliche Grö$\mathrm{Be}$, Anzahl und Dauer von Mischwasserentlastungen zu berechnen. Figur 6 zeigt als Ergebnis einer Simulation die statistische Verteilung der Entlastungsereignisse eines Überlaufs der Kanalisation in der Gemeinde Fehraltorf bei Zürich für vier Planungsszenarios. Betrachtet werden hier die maximalen Einleitungsraten, die für die lokalen ökologischen Verhältnisse im Gewässer von Bedeutung sind (vergleiche Tabelle 3). Dargestellt ist das Entlastungsverhalten des bestehenden Mischsystems mit Becken ("MS 


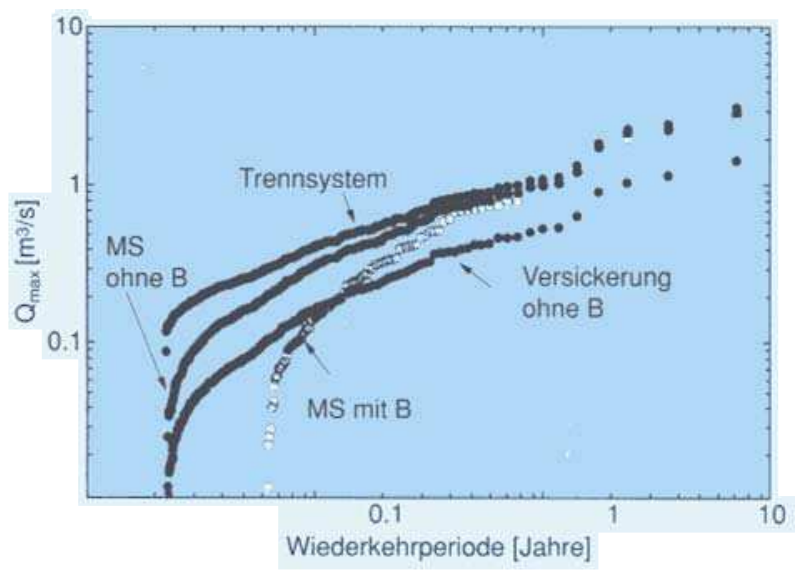

Tabelle 3. Mögliche ökologische Auswirkungen von Mischwassereinleitungen in Fließgewässern.

\begin{tabular}{ll}
\hline Ursachen & Akute Wirkungen (in Stunden) \\
\hline Plötzliche Zunahme der Fließgeschwindigkeit & $\begin{array}{l}\text { Erhöhte Drift von Organismen, welche die Ge- } \\
\text { wässersohle bewohnen (Benthosorganismen) }\end{array}$
\end{tabular}

Entstehung von Geschiebetrieb

Eintrag sauerstoffzehrender Substanzen

Eintrag von giftigen (toxischen) Substanzen (zum Beispiel Ammoniak)

Eintrag von toxischen Substanzen (z.B. Nitrit)

Sedimentation organischer Feststoffe

Eintrag pathogener Keime

Sedimentation von Feststoffen

Eintrag von schwer abbaubaren organischen Stoffen und Schwermetallen

Eintrag von Nährstoffen (Phosphat)
Figur 6. Statistische Verteilung simulierter Überlaufereignisse: Mischwasserentlastungen aus einem Überlaufbecken in der Kanalisation Fehraltorf (Schweiz) für die Sommer der Jahre 1982-1988 bei vier Ausbauvarianten: Mischsystem mit heutigem Mischwasserbecken ("MS mit B"), Mischsystem ohne Becken ("MS ohne B"), Versickerung aller Dachabflüsse ohne Becken, Trennsystem. Die Abszisse gibt an, in welcher Zeitspanne Überlaufereignisse durchschnittlich wiederkehren, auf der Ordinate findet man die dazugehörige maximale Abflußrate $\left(Q_{\max }\right)$ der Abwassereinleitung.

mit B") im Vergleich zu den Varianten ohne Mischwasserbecken ("MS ohne B"), für den Fall einer Versickerung aller Dachabflüsse sowie für den Fall eines Trennsystems. Daraus wird deutlich, daß durch die Wirkung der Becken die Anzahl der kleinen Entlastungen abnimmt; die Häufigkeit großer Abflüsse hängt davon jedoch nur unwesentlich ab. Die Versickerung aller Dachabwässer würde zu einer Reduzierung der maximalen Entlastungsabflüsse bei seltenen Spitzenereignissen etwa um die Hälfte führen. Ein Trennsystem brächte hingegen noch höhere Abflüsse, da sämtliches Regenwasser lokal in das Gewässer eingeleitet wird.

Über Mischwasserentlastungen können in kurzer Zeit erhebliche Schmutzstofffrachten in die Vorfluter gelangen. Ammonium (oder Ammoniak) ist ein typischer Bestandteil häuslichen $\mathrm{Ab}$ wassers und eignet sich deshalb als Indikator für den Anteil häuslichen Schmutzwassers im Mischwasser. Wie die Abflußmenge (in $\mathrm{m}^{3} / \mathrm{s}$ ) kann mit Modellrechnungen auch die Schmutzbelastung des Vorfluters während eines Überlaufs ermittelt werden (zum Beispiel die Konzentration von Ammoniak in $\mathrm{mg} / \mathrm{L}$ ). Im Falle des Überlaufs der Kanalisation in Fehraltorf (vergleiche Figur 6) ist ein Ergebnis für das Szenario "MS mit B", daß durch Mischwasserbecken lediglich die Häufigkeit zeitweise erhöhter Ammoniakkonzentrationen im Vorfluter vermindert wird (Figur 7). Interessanterweise sind jedoch weder die Spitzenkonzentrationen noch die maximalen Einwirkzeiten bei Entlastungsereignissen deutlich geringer.

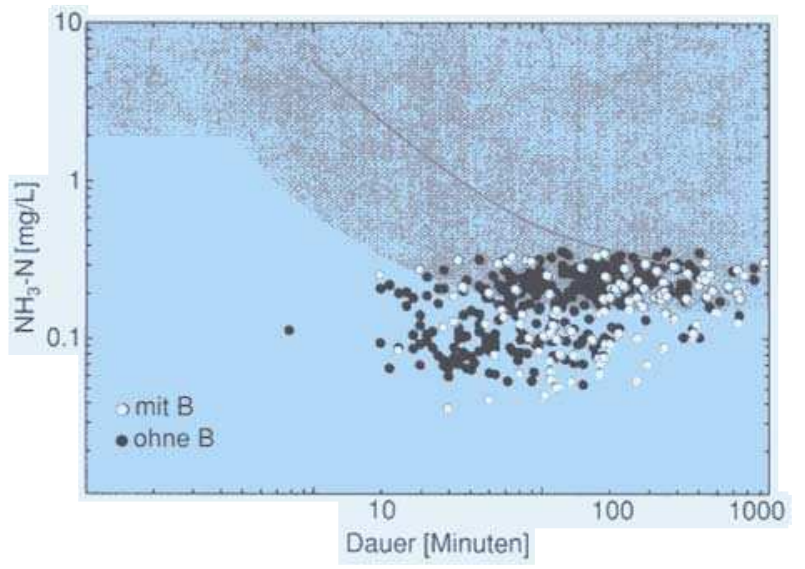

Figur 7. Ammoniakkonzentration in der Luppmen bei Fehraltorf, ermittelt durch eine Langzeitsimulation der Regenereignisse im Sommer der Jahre 1982-1988. Vergleich der Varianten mit und ohne Mischwasserbecken ("mit B" bzw. "ohne B"). Der dunkle Bereich kennzeichnet bei unterschiedlichen Einwirkzeiten diejenigen Ammoniakkonzentrationen (Zusammenfassung von in der Literatur vorgeschlagenen - eine Pionierarbeit [19] ist durch die eingezeichnete Linie hervorgehoben - Grenzwerten [20]), bei denen sich Schädigungen an Fischen nachweisen lassen. Die Konzentrationen wurden unter folgenden Annahmen für die Verhältnisse in der Luppmen berechnet: Temperatur $20^{\circ} \mathrm{C}$, Durchfluß 30 Liter pro Sekunde, pH-Wert 8.5, Ammoniakkonzentration vor der Einleitung $\mathrm{NH}_{3}-\mathrm{N}=$ $0 \mathrm{mg} / \mathrm{L}$, spezifisches Speichervolumen der Mischwasserbecken 30 Kubikmeter pro Hektar (heutige Beckengröße, bezogen auf die versiegelte Fläche in Hektar). 


\author{
Entstehung und Transport von \\ Mischwasserabflüssen in \\ Entwässerungssystemen sind heute \\ prinzipiell bekannt und mit meist \\ hinreichender Genauigkeit quantifizierbar. \\ Numerische Prozeßmodelle bauen auf \\ diesen Kenntnisstand auf und ermöglichen \\ Prognosen über die Auswirkungen \\ technischer Maßnahmen im \\ Entwässerungssystem.
}

\section{Welche ökologischen Wirkungen haben Mischwassereinleitungen?}

Mischwassereinleitungen verursachen stoßartige Belastungen der Gewässer durch schnell steigende Abflüsse und Stofffrachten. Letztere stammen aus dem Schmutzwasser, aus Abschwemmungen von Siedlungsflächen und aus Ablagerungen in den Kanälen. Tabelle 3 gibt eine Übersicht über die nach derzeitigem Wissensstand zu erwartenden ökologischen Wirkungen. größeren zeitlichen und räumlichen Grenzen bemerkbar sind.

Plötzlich erhöhte Abflüsse und der damit einhergehende Geschiebetransport können das Abdriften von Gewässerorganismen verursachen. Bei häufiger Wiederholung droht eine Verarmung der Biozönose in dem betroffenen Gewässerabschnitt. Als Maß für den eventuellen Schaden gelten die Art und Zahl der verdrifteten Organismen und die Häufigkeit sowie die räumliche Ausdehnung der Verdriftung. Bei der Beurteilung spielt die Gewässermorphologie eine sehr wichtige Rolle. Die negative Wirkung erhöhter Abflüisse wird entscheidend von sogenannten Refugialräumen kompensiert. Das sind strömungsruhige Zonen an der Sohle, in den seitlichen Rändern und vor allem im hyporheischen Interstitial (Hyporheal wird das Porenund Lückensystem unterhalb der Gewässersohle genannt).

Akute Schadwirkungen des überlaufenden Mischwassers werden vor allem durch toxische Stoffe (besonders Ammoniak) verursacht. Durch sauerstoffzehrende Stoffe im eingeleiteten Abwasser kann gleichzeitig der Sauerstoffgehalt im

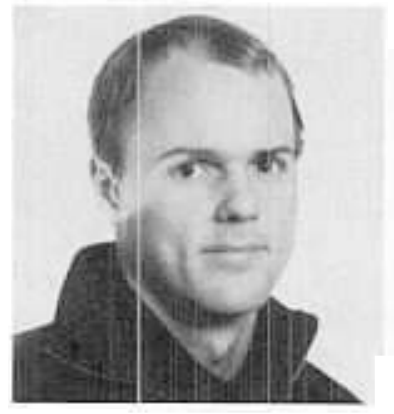

Jorg Lange: Getoren 1963 in Darmstadt, Hassen. Studium der Biologie an der Technischen Hochschule Damstadt und der Universitat Freiburg im Breisgau mit Schwerpunkt Limnologio. Diplom 1990. Seit 1993 Mitarbeit im Arbeitskreis Berutsbild und Selbstverständnis in der Biologie (AkBuSiB e.V.), Forschungsarbeiten zur Ókotogie des Rheins und zur Geschichte der Limnologie, Seit 1991 Mitarbeit am Proiekt "Gewässerschut: bei Regenwetter" der Eidgenossischen Anstalt fur Wasserversargung, Abwasserreinigung und Gewässerschutz (EAWAG); zur Zeit freier Autor eines Buchs uber den Rhein.

Für die akuten und verzögerten Wirkungen von Mischwassereinleitungen in Fließgewässern ist das Verhältnis von Mischwasserabfluß zu Gewässerabfluß zur Zeit der Einleitung maßgebend. Wesentlich für die ökologische Bewertung der akuten und verzögerten Wirkungen ist die Tatsache, daß die kritischen physikalischen und stoffspezifischen Parameter simultane Vorgänge kennzeichnen (zum Beispiel die Bewegung der Gewässersohle und die toxische Wirkung von Ammoniak bei gleichzeitigem Sauerstoffdefizit).

Während die akuten und verzögerten Schädigungen im Zeitmaß von Stunden und in relativ kurzen Gewässerabschnitten verlaufen, manifestieren sich die Langzeitwirkungen durch Schädigungen, die wegen der Akkumulation von verschiedenen Stoffen in weitaus
Gewässer verringert werden. Die Folge sind oft synergistische Effekte, also eine Verstärkung durch die Überlagerung mehrer negativer Einflüsse. Schädigungen von Organismen können also bereits dann eintreten, wenn die Einzelursachen (jeweils für sich allein betrachtet) noch gar keine kritische Größe erreichen.

Ökologische Langzeitwirkungen resultieren aus einer Vielzahl von Entlastungsereignissen und der Akkumulation von Stoffen in Organismen. Bei großen Fließgewässern beeinflussen die vielen und zu unterschiedlichen Zeiten erfolgten Mischwasserentlastungen auch den Gewässerzustand bei Trockenwetter maßgebend. Dies gilt auch für die Seen. Zu den Langzeitwirkungen gehört zum Beipiel die Akkumulation von Schwermetallen in Sedimenten großer und langsam strömender Flüsse. In Seen werden Eutrophierungsprobleme durch die im Mischwasser enthaltenen Nährstoffe verstärkt

Bei einer Fallstudie in der Luppmen, einem Bach bei Fehraltorf im Kanton Zürich, werden die Auswirkungen von Mischwassereinleitungen auf die Benthos-Biozönose, die Lebensgemeinschaft von Pflanzen und Tieren auf der Gewässersohle, untersucht. Durch Sauerstoffmessungen sowie die sensorische Begutachtung (Geruch, Farbe) unterhalb des Überlaufs ist nachzuweisen, daß sich durch Mischwasser eingetragenes, organisches Material bei geringen Fließgeschwindigkeiten lokal im Bachbett absetzt und dort vor allem im Hyporheal eine erhöhte Sauerstoffzehrung hervorruft (Figur 8). Erst einige Tage nach einem Mischwasserüberlauf stellen sich die bei Trockenwetter üblichen Sauerstoffkonzentrationen wieder ein.

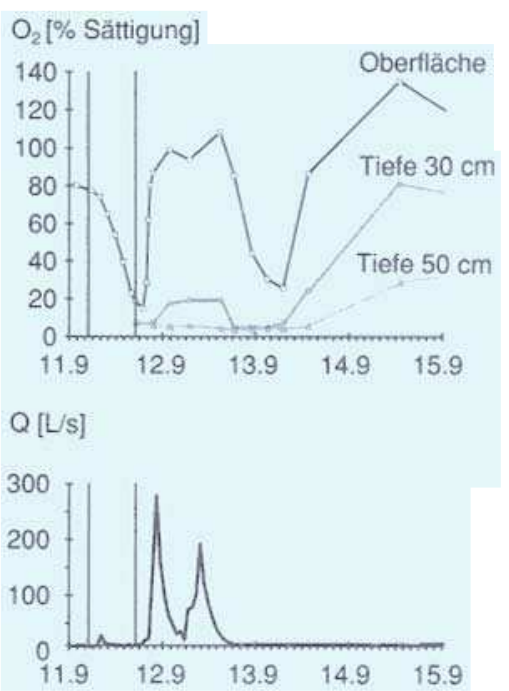

Figur 8. Sauerstoffsättigung $\left(\mathrm{O}_{2}\right)$ in der Gewässersohle (hyporheisches Interstitial) nach Mischwasserentlastungen. Infolge einer hochkonzentrierten Einleitung am 11. September um ca. 7 Uhr in den ausgetrockneten Bach fällt die Sauerstoffsättigung im freien Wasser bis unterhalb $20 \%$, $50 \mathrm{~cm}$ tief im Interstitial auf weniger als $5 \%$. Gegen 18 Uhr bewirkt eine zweite Entlastung mit hohem Abfluß, aber geringer Stoffkonzentration wieder einen Anstieg der Sauerstoffsättigung. Erst später folgt die Hochwasserwelle aus dem natürlichen Einz'ugsgebiet; im Interstitial steigt die Sauerstoffsättigung dabei nur geringfügig an. Mit dem Rückgang des Durchflusses im Bach nimmt die Sauerstoffsättigung (12. 9., ca. 18 Uhr) infolge Zehrung durch Abbau organischer Stoffe wieder ab. Nach mehr als zwei Tagen pendelt sich die Sauerstoffsättigung sowohl an der Oberfläche wie auch im Interstitial auf die bei Trockenwetter üblichen Werte ein. 


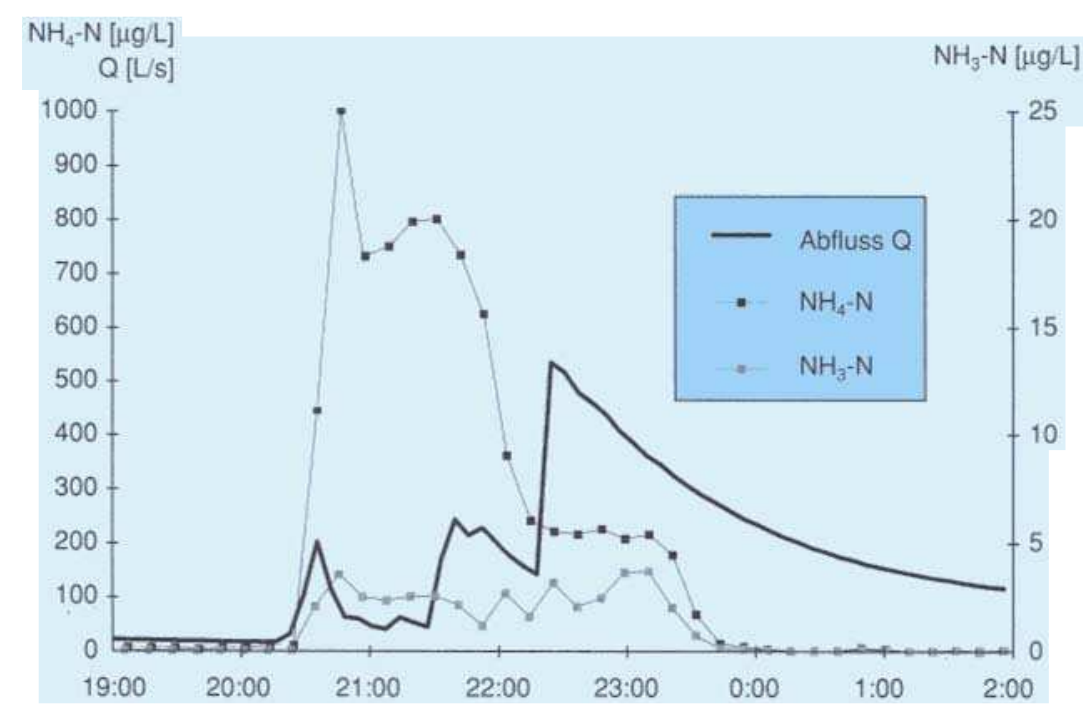

Figur 9. Konzentrationen von Ammonium- $\left(\mathrm{NH}_{4}^{+}\right)$und Ammoniak $\left(\mathrm{NH}_{3}\right)$ bei einem Mischwasserüberlauf im Bach Luppmen bei Fehraltorf nach einem Entlastungsereignis im Juli. Bei Trockenwetter ist Ammonium in Basisabfluß kaum nachweisbar $\left(T=12^{\circ} \mathrm{C}, \mathrm{pH} \approx 7.0\right)$. Mit Beginn des Überlaufvorgangs $(20.30 \mathrm{~h})$ steigt gleichzeitig die Ammoniumkonzentration. Entsprechend der Temperatur $\left(T=15^{\circ} \mathrm{C}\right)$ und des $\mathrm{pH}$-Werts $(\mathrm{pH} \approx 7.2)$ bleibt die Ammoniakkonzentration zunächst relativ niedrig; sie nimmt erst mit dem Eintreffen des relativ warmen Bachwassers aus dem natürlichen Einzugsgebiet etwa zwei Stunden später $\left(T=17^{\circ} \mathrm{C}, \mathrm{pH} \approx 8.0\right) \mathrm{zu}$.

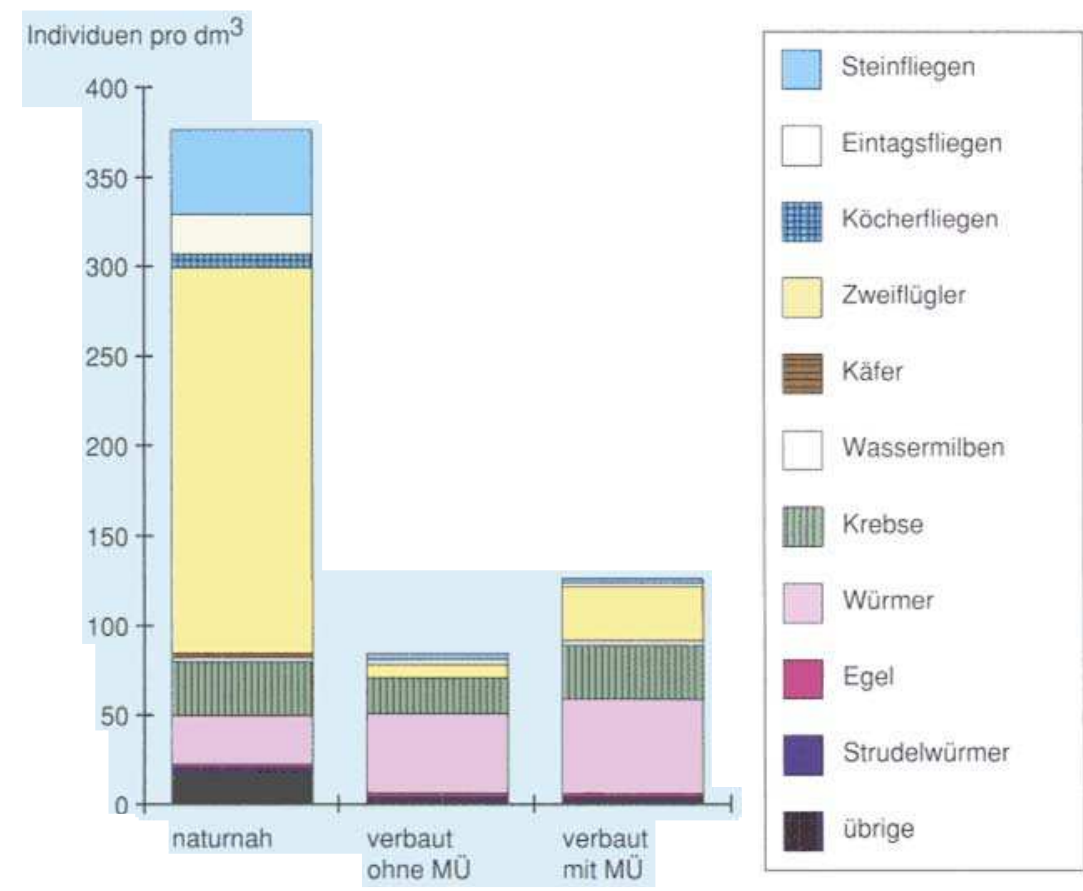

Figur 10. Die Besiedlung der Luppmen bei Fehraltorf im Sommer: Makroinvertebraten in der Bach:sohle an drei Beobachtungsstellen, "naturnah" in der unverbauten Strecke (Referenz), die anderen in der verbauten Strecke. Die Höhe der Balken gibt die Individuenzahlen an, die Balkenabschnitte kennzeichnen die Taxazugehörigkeit. In den verbauten Bachstrecken verschwinden Eintags-, Köcher- und Steinfliegenlarven gegenüber Organismen, die weniger hohe Ansprüche bezüglich der physikalischen und morphologischen Bedingungen stellen (Krebse, Würmer), Die Gleichförmigkeit der Gewässergestaltung, die hohen Wassertemperaturen und geringen Fließgeschwindigkeiten bei Trockenwetter spiegeln sich auch in der relativen Gieichförmigkeit der Besiedlung wieder. Die Besiedlung des von Mischwassereinleitungen betroffenen Abschnitts "mit Mü" unterscheidet sich nur wenig von der des nicht durch Mischwasser beeinträchtigten, jedoch verbauten Abschnitts ("ohne Mü").
Ammonium $\left(\mathrm{NH}_{4}^{+}\right)$stammt vorwiegend aus Abwassereinleitungen. Seine Konzentration steht in einem von der Wassertemperatur und dem $\mathrm{pH}-$ Wert abhängigen chemischen Gleichgewicht mit der Konzentration des für Organismen giftigen Ammoniaks $\left(\mathrm{NH}_{3}\right)$. Oft steht eine erhöhte Ammoniakkonzentration im Verdacht, Ursache für Fischsterben in unseren Bächen zu sein (etwa infolge Einleitungen von Gülle). Es liegt auch aus diesem Grund nahe, die Konzentration dieser Stickstoffverbindungen $\mathrm{zu}$ erfassen, um somit Auswirkungen auf die Gewässerbiozönose abschätzen zu können. Figur 9 zeigt den gemessenen Verlauf der Ammonium- und Ammoniakkonzentrationen im Bach Luppmen bei Fehraltorf nach einem Entlastungsereignis, bei dem allerdings keine für Fische toxische Konzentrationen erreicht worden sind.

Mit Schmutzstoffen als Nahrungsangebot vermehren sich im Gewässer vorwiegend solche Mikroorganismen, die unter Sauerstoffverbrauch diese Stoffe mineralisieren (Selbstreinigung). Die mineralisierten Abbauprodukte werden dann in einer Nahrungskette von Pflanzen und Tieren aufgenommen.

Wie die Untersuchung der Kleintierwelt in der Luppmen zeigt, unterscheidet sich die Besiedlung der Bachsohle und des Hyporheals im nicht verbauten, naturnahen Abschnitt erheblich von der in befestigten Abschnitten weiter unterhalb (Figur 10). Die unterste Bobachtungsstelle ("verbaut mit MÜ") ist durch Mischwassereinleitungen betroffen. Dennoch unterscheidet sich die Artenzusammensetzung an dieser Stelle kaum von derjenigen an den weiter oben liegenden, durch Mischwasser nicht beeinflußten Stellen. Die beobachteten Unterschiede lassen sich vor allem auf die morphologische Qualität und das unterschiedliche Abflußregime der drei Gewässerabschnitte zurückführen. Eintags-, Köcher- und Steinfliegenlarven stellen hohe Ansprüche an Sohlenbeschaffenheit, Strömung, Temperatur und Sauerstoffgehalt. Sie verschwinden deshalb im ausgebauten Bereich, wo das Wasser zeitweise kaum fließt, sich im Sommer stark erwärmt und lokal sogar austrocknet. Dort kommen vermehrt solche Organismen vor, die an Bedingungen wie niedriger Sauerstoffgehalt und geringe Sohlenheterogenität angepaßt sind (Krebse und Würmer).

Obwohl also Mischwassereinleitungen erhebliche Veränderungen des Stoffhaushalts im Gewässer hervorrufen können, überwiegt die Auswirkung anderer Störfaktoren wie ungünstige 
morphologische Beschaffenheit und Sohlenbefestigung auf die Bachbiozönose in der Luppmen. Mit anderen Worten: Eine stark durch Ausbaumaßnahmen beeinträchtigte Gewässerbiozönose wird durch Mischwassereinleitungen kaum zusätzlich geschädigt.

\section{Veränderungen von \\ Lebensgemeinschaften in Gewässern durch Mischwassereinleitungen werden zwar postuliert, können aber (heute nochı) nicht eindeutig nachgewiesen werden. Ökologisch begründete Toleranzgrenzen für Einleitungsmengen und -konzentrationen von Mischwasser sind daher nur schwer festzulegen.}

\section{Wie sieht die neue Entwässerungsstrategie aus?}

Die zukünftige Strategie zum Schutz von Gewässern in Siedlungsgebieten wird das gesamte Wasser- und Stofftransportsystem berücksichtigen: die Quellen der Verunreinigungen, den Transport, die Behandlung des Abwassers und das Gewässer als ökologischen Lebensraum. Ähnlich wie heute schon bei Kläranlagen werden auch die Maßnahmen im Entwässerungsnetz differenzierter geplant werden. Mit Simulationsmodellen wird der Ingenieur die Menge und Zusammensetzung von Mischwasserfrachten genügend genau voraussagen können. Somit kann der Effekt von Gewässerschutzmaßnahmen auch lokalspezifisch abgeschätzt werden. Allerdings ist der Zusammenhang zwischen den relativ genau beschreibbaren Einleitungen und den ökologischen Reaktionen des Gewässers noch zu wenig erforscht ${ }^{[14]}$.

Aus den bisher vorliegenden Befunden und Erkenntnissen ergeben sich aber bereits Hinweise für eine bessere $\mathrm{Ge}$ wässerschutzstrategie bei Regenwetter: 1. Angesichts der bisher noch unklaren Beziehungen zwischen Mischwassereinleitung und ökologischer Auswirkung sind "fehlerfreundliche" (überschaubare, zeitlich abgestufte) Maßnahmen zum Schutze der Gewässer anzuraten (Mischwasserbecken an jedem Überlauf sind nicht fehlerfreundlich).

2. Solange die Ursache-Wirkung-Beziehungen ungeklärt sind, sollten Maßnahmen im Entwässerungssystem und im Gewässer ökologische Vielfalt fördern, also Lebensgemeinschaften ermöglichen, die sich in weitgehend naturbelassenen Gewässern auch einstellen würden.

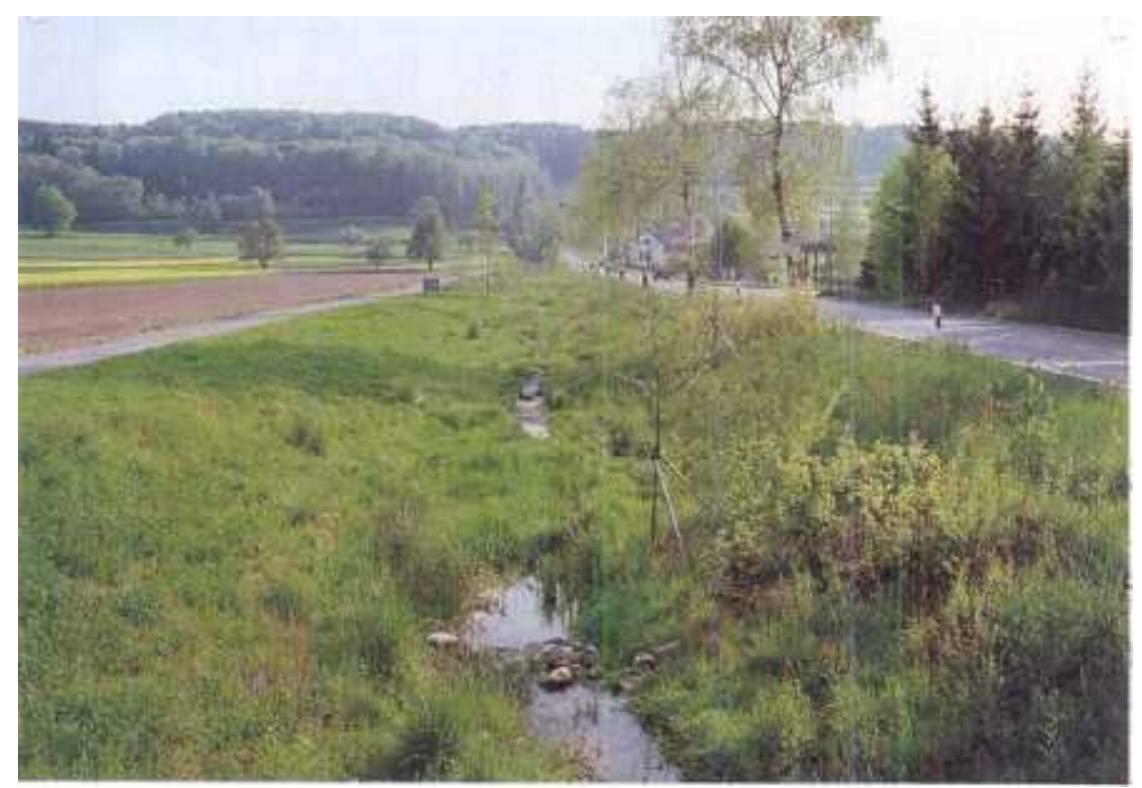

Figur 11. Der renaturierte Chämmeterbach in Dübendorf bei Zürich. Im nach wie vor erhaltenen Hochwasserbett mäandert der Bach mit variierendem Fließquerschnitt und umfließt verschiedene Hindernisse mit sehr unterschiedlichen Fließgeschwindigkeiten. Die Böschungen sind stark abgeflacht. Gegenüber dem vorherigen Zustand als Entwässerungsgraben braucht der Bach nun ein Mehrfaches an Grundfläche (aufgenommen 1990, zwei Jahre nach Beendigung der Umgestaltung).
3. Entwässerungstechnische Maßnahmen sollen zusammen mit einer Ge-wässerRevitalisierung geplant werden, die sich an eine ökologisch verträgliche Nutzung des Gewässers anlehnt, also extreme Eingriffe in den Gewässerlauf und seine Wasserführung unterläßt (vergleiche Figur 11).

4. Die heute vollkommene und schnelle Abwasserableitung aus Siedlungsgebieten werden aufgegeben. Stattdessen wird verunreinigtes und wenig verunreinigtes Abwasser konsequent getrennt. Das wenig verunreinigte Abwasser kann versickern.

5. Mit moderner Technik (Simulationsmodelle, Abflußsteuerung et cetera) wird sowohl bei der Planung als auch im Be- trieb städtischer Entwässerungssysteme gewährleistet, daß die Mindestanforderungen für Abwassereinleitungen auch tatsächlich eingehalten werden.

Die neue Entwässerungsstrategie beabsichtigt, in künftig revitalisierte Gewässer

Regenwasser aus Siedlungen nur dann einzuleiten, wenn es nicht versickert werden kann,

Abwasser am Ort der Entstehung zu vermeiden,

unterschiedlich verschmutztes Abwasser nicht zu mischen und den

Regenwasserabfluß zu verzögern.

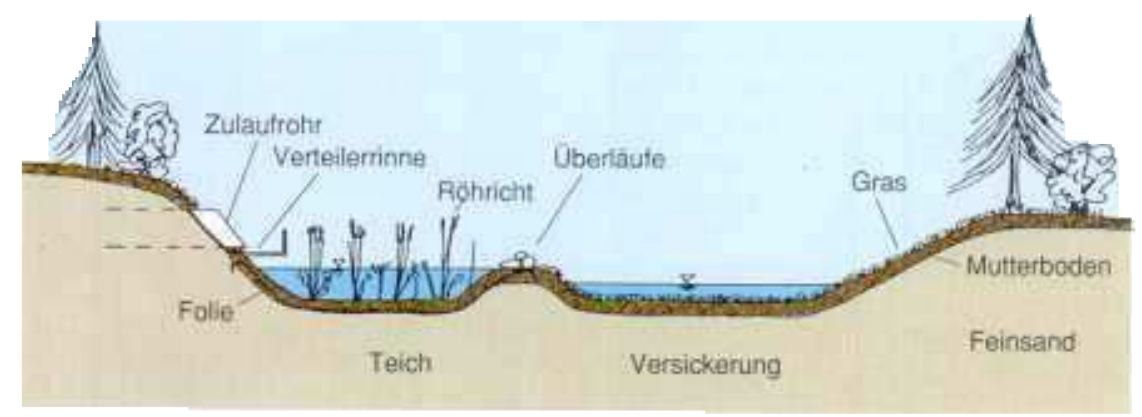

Figur 12. Regenwasserversickerung mit vorgeschaltetem Teich. Das Regenwasser aus einer kleinen Überbauung wird über eine Verteilerrinne in einen Teich geleitet. Der Teich soll die Fließgeschwindigkeit reduzieren und dadurch die Sedimentation von Feststoffen begünstigen. Er ist hier mit einer Folie abgedichtet und kann mit Röhricht oder anderen Makrophyten bepflanzt sein, wodurch Feststoffe noch besser zurückgehalten werden. Der Überlauf des Teichs mündet in eine begrünte Mulde, in der das Regenwasser versickert. 
Korrekturen zur naturnahen Gestaltung und Revitalisierung der kleinen Fließgewässer sind zwar vor allem wegen des Flächenbedarfs teuer (Figur 11), erscheinen heute aber politisch sowie finanziell machbar. Aus einem Bericht über das "Wiederbelebungsprogramm für Fließgewässer im Kanton Zürich" [15] geht hervor, daß von den $563 \mathrm{~km}$ Fließgewässern $112 \mathrm{~km}$ verdolt sind. Mit wenigen Ausnahmen seien diese Gewässer als wiederbelebungsbedürftig anzusehen. In der Stadt Zürich werden Bachöffnungen und Revitalisierungen in einer Gesamtlänge von $55 \mathrm{~km}$ für realisierbar erachtet ${ }^{[16]}$.

Modifikationen des technischen Entwässerungssystems sind sowohl bei den privaten Entwässerungsanlagen (Liegenschaftsentwässerung) als auch bei den öffentlichen Anlagen (Ortsentwässerung) notwendig. Für die unerläßliche Koordination von Liegenschafts- und Ortsentwässerung ist in der Schweiz durch die neuen Richtlinien gesorgt ${ }^{[11,17]}$. Ihre Durchsetzung ist allerdings mit groBem Schwierigkeiten bis hin zum Widerstand der Beteiligten verbunden, weil die Maßnahmen bis in die unmittelbare Privatsphäre des einzelnen Bürgers reichen.

Wirkungsvoll und relativ leicht in die Tat umzusetzen ist die Versickerung und Retention des Regenwassers auf dem jeweiligen Grundstiick oder in der nahen Umgebung. Unbequem bei Regenwetter sind unbefestigte Wege oder Rasengittersteine auf Parkplätzen. $\mathrm{Zu}$ den teuersten und am schwierigsten realisierbaren Maßnahmen gehört die Abtrennung der bestehenden Sickerwasserleitungen vom Anschluß an das übrige Entwässerungsnetz.

Die Schwerpunkte der Korrekturen an der Ortsentwässerung sind einerseits die Abtrennung der ans Entwässerungsnetz angeschlossenen Bäche (wenn möglich durch Offenlegung und $\mathrm{Re}$ vitalisierung) und anderseits die bessere Ausnützung der vorhandenen Retentionsmöglichkeiten im Entwässerungsnetz durch laufende und gezielte Eingriffe in den Entwässerungsvorgang (Abflußsteuerung). Daneben soll auch das von öffentlichen Plätzen und Straßen abfließende Regenwasser soweit wie möglich von der Kanalisation ferngehalten werden. In Figur 12 und Figur $13^{[18]}$ sind nur zwei von einer Vielzahl möglicher Maßnahmen dargestellt.

Nach der Realisierung aller dieser Maßnahmen werden Mischwasserentlastungen wesentlich seltener vorkommen. Technische Maßnahmen zur Behandlung des Mischwassers wie Misch-

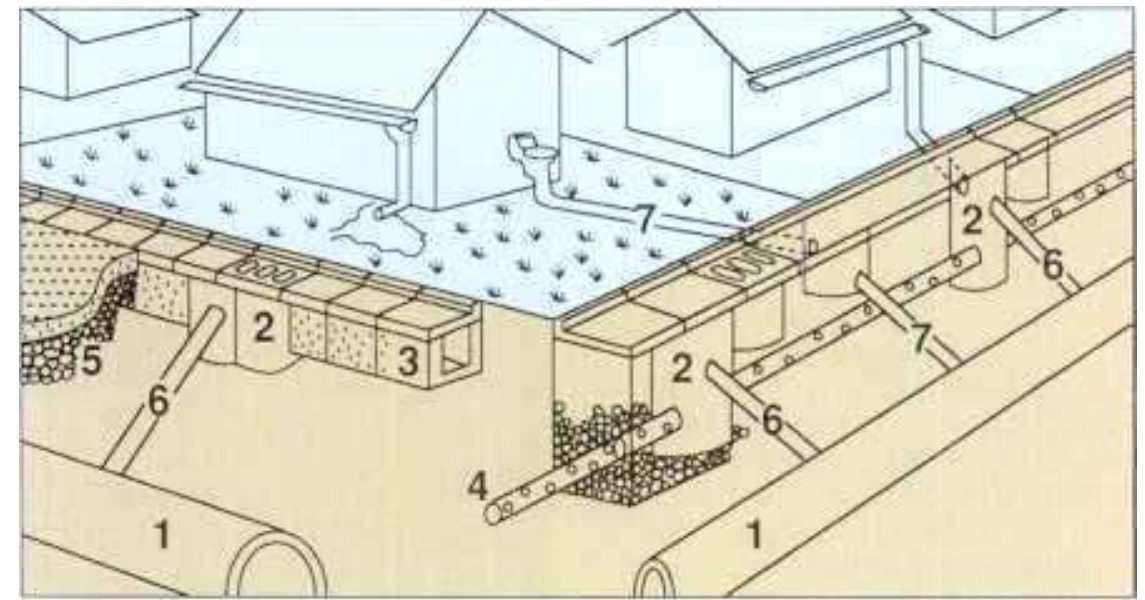

Figur 13. Entwässerungsschema des experimentellen Einzugsgebietes in Tokio. Der Abfluß von Dächern und Straßen wird durch Versickerung in Schächten reduziert. Die Sickerschächte (2) sind durch Sickerrinnen (3) und Sickerleitungen (4) miteinander verbunden. Bei ungenügender Sickerleistung wird das Regenwasser über den Überlauf des Sickerschachtes (6) in die Mischwasserkanalisation (1) geleitet. Weiterhin vermindern durchlässige Beläge (5) den Regenwasserabfluß. Schmutzwasserleitungen (7) sind nach wie vor an die Mischkanalisation angeschlossen.

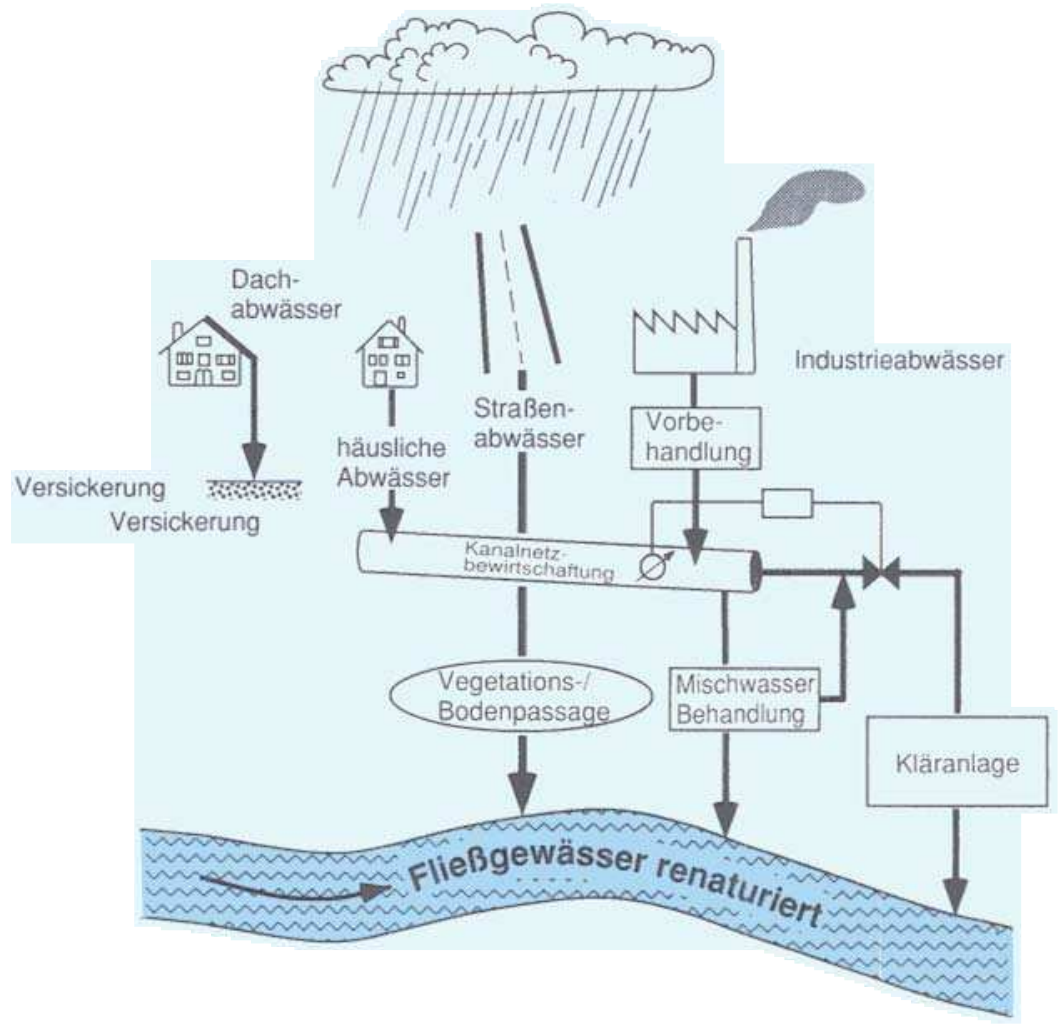

Figur 14. Das zukünftige Entwässerungssystem. Eine ökologisch und ökonomisch bewußte Siedlungsentwässerung vermeidet die schnelle Abwasserableitung und die Mischung von wenig und stark verschmutztem Abwasser: Dachwässer werden versickert, Straßenabwässer naturnah gereinigt und langsam abgeleitet. Nur stärker verschmutzte Abwässer werden zu einer mehrstufigen Kläranlage geführt (Phosphor- und Stickstoffelimination, Filtration). Bei Regenwetter sorgt ein Bewirtschaftungssystem dafür, daß erst nach vollständiger Aktivierung des gesamten Speicherraums Mischwasserentlastungen auftreten. Diese werden nach den gewässerspezifischen Erfordernissen weiter behandelt (Sedimentation, Siebung etc.), bevor der Überlauf in ein naturnah revitalisiertes und ökologisch verträglich genutztes Gewässer eingeleitet wird. 
wasserbecken, Wirbelabscheider, Rechen und so weiter werden lokalspezifisch eingesetzt und oft miteinander kombiniert. In Figur 14 sind die Elemente zukünftiger Siedlungsentwässerung skizziert.

Siedlungs- und Verkehrsflächen in der Schweiz, die neue Entwässerungsanlagen brauchen, nehmen immer noch um etwa $2 \%$ pro Jahr zu. Darüber hinaus ist das bestehende Entwässerungsnetz zu erneuern. Bei einer geschätzten Lebensdauer der Kanalisation von 50 bis 80 Jahren beträgt die Erneuerungsrate 1.5 bis $2 \%$ pro Jahr. Der Zuwachs von erneuerten Entwässerungsanlagen wird somit in 25 Jahren auf 30 bis $40 \%$ geschätzt. Daß heißt, daß mindestens $50 \%$ der Entwässerungsanlagen im Jahre 2015 dem angestrebten Entwässerungskonzept entsprechen können, sofern die neue Strategie jetzt und umfassend angewendet wird.

Die ersten Korrekturen sind heute erfolgreich realisiert. Dennoch kann man nicht behaupten, daß sich die neue Entwässerungsstrategie bereits überall durchgesetzt hat. Vor allem seitens der Gemeindeverwaltungen und der Politiker gibt es Bedenken; sie ziehen den Bau einer neuen Schule oder Straße der Kanalisation vor, zumal wenn diese schon existiert und einer zufriedenen Mehrheit keinen AnlaB zu Beschwerden gibt. Weitere Fortschritte des Gewässerschutzes bei Regenwetter sind auf eine bessere Information der Öffentlichkeit über diese Problematik angewiesen.

\section{Die zukünftige Siedlungsentwässerung ist mit steigenden Kosten und Einschränkungen beim Komfort verbunden. \\ Ohne breite Akzeptanz wird sich die neue Strategie nicht durchsetzen, die heute noch lösbaren \\ Probleme des Gewässerschutzes könnten sich bald verschärfen.}

\section{Literaturverzeichnis}

[1] M. Illi: Von der Schissgruob zur modernen Stadtentwässerung (herausgegeben von de Stadtentwässerung Zürich), Verlag Neue Zürcher Zeitung, Zürich (1987), p. 263.

[2] ATV: Lehr- und Handbuch der Abwassertechnik, 3. Auflage, Band II: Entwurf und Bau von Kanalisationen und Abwasserpumpwerken. Ernst \& Sohn, Berlin (1982).

[3] G. Garbrecht: Wasser - Vorrat, Bedarf und Nutzung in Geschichte und Gegenwart, rororo 7724, Rowohlt, Reinbek (1985), p. 279.
[4] H. Brombach: "Häufigkeit und Verteilung der Kanalisationsverfahren in Deutschland", Korrespondenz Abwasser, im Druck.

[5] W. Gujer: "Entwässerungsanlagen müssen betrieben, unterhalten und erneuert werden", Manuskript, VGL/VSA-Tagung "Neue Anforderungen an Gemeinden im Gewässerschutz" (18.September 1990)

[6] Verordnung über Abwassereinleitungen, Schweizerischer Bundesrat, Bern (8.12.1975).

[7] W. Schilling: "Regenwasserbehandlung im Ausland - Übersicht zum Stand der Technik in einigen Industrieländern", Mitteilungen des Instituts für Wasserwirtschaft der Universität Hannover 60 (1986) 89-107.

[8] A. Hörler: Ingenieur-Handbuch, 78. Auflage Band II: Kanalisation, Schweizer Verlagshaus AG, Zürich (1966).

[9] EAWAG: Regionale abwassertechnische Studie Glattal, Teile I-III, Dübendorf (1979).

[10] W. Gujer, V. Krejci: "Kenntnisse und Forschung auf dem Gebiet der Regenwasserbehandlung", in: VSA-Verbandsbericht Nr. 142/4, Verband Schweizerischer Abwasserfachleute, Zürich (1979)

[11] Genereller Entwässerungsplan (GEP), Verband Schweizerischer Abwasserfachleute, Zürich (1989)

[12] ATV: Arbeitsblatt A 128: Richtlinien für die Bemessung und Gestaltung von Regenentlastungen in Mischwasserkanälen, Gesellschaft zur Förderung der Abwassertechnik (GFA), (1977) 1-50.

[13] ATV: "Beeinflußung der Beschaffenheit von Fließgewässern durch Mischwassereinleitungen. Ergebnis einer Anhörung von

Fachleuten am 18. und 19. April 1988 in Essen", Korrespondenz Abwasser 36/7 (1989) 755-760.

14] J. Lange, S. Gammeter, V. Krejci, W. Schilling: "Gewässerschutz bei Regenwetter - Fallstudie Fehraltorf / ZH", Mitteilungen der EAWAG Nr. 32D (1991) $18-23$.

[15] H. P. Willi, C. Göldi, G. Keller: "Kanton Zürich: Wiederbelebungsprogramm für die Fließgewässer - Bericht August 1988", Gas Wasser Abwasser 69 (1989) 670-686.

[16] F. Conradin, U. Räbsamen, J. Villiger: "Das Bachkonzept: Anlaß, Ziel, Umfang", Gas Wasser Abwasser 68 (1988) 393-398.

[17] Schweizer Norm SN 592000: Planung und Erstellung von Anlagen für die Liegenschafts entwässerung, Verband Schweizerischer Abwasserfachleute und Schweizerischer Spenglermeister- und Installateur-Verband, Zürich (1990).

[18] S. Fujita: "Experimental Sewer System, its Application and Effects", Proceedings of the 4th International Conference on Urban Storm Drainage, Lausanne (1987).

[19] K. Wuhrmann, H. Woker: "Experimentelle Untersuchungen über die Ammoniak- und Blausäurevergiftung (Beitrag zur Fischtoxikologie)", Schweizische Zeitschrift für Hydrolologie 15 (1949) 236-260.

[20] K. Whitelaw, J. F. de L. G. Solbe : "River Catchment Management: An Approach to thi Derivation of Quality Standards for Farm Pollution and Strom Sewage Discharges", Water Science and Technology 21 (1989) 1065-1076.

(Eingegangen am 27. November 1991 mitgeteilt von W. Giger

\section{$\square$ Anmerkung des zuständigen Mitherausgebers:}

Im Beitrag von Krejci, Lange und Schilling wird ein für die Gegenwart und Zukunft des Gewässerschutzes bedeutsamer Problemkomplex angeschnitten, wobei die Blickrichtung der Ingenieure überwiegt; es wird dabei aber klar, wie notwendig für den Gewässerschutz bei Regenwetter neue Sichtweisen sind, die technisch-konstruktive und ökologische Aspekte miteinander verknüpfen. Gefragt ist somit interdisziplinäre Zusammenarbeit von Ingenieuren und ökologisch ausgerichteten Umweltnaturwissenschaftlern (Biologen, Chemikern). Für die Biologen ergibt sich hieraus die Schwierigkeit, daß die Effekte stoßartiger Belastungen schlecht von anderen Einwirkungen unterschieden werden können. Diese Probleme werden erst seit kurzem in einer Art und Weise bearbeitet, welche über die phänomenologische Beschreibung hinausgeht und nach kausalen Zusammenhängen sucht. In einem kommenden GAIAArtikel werden Fließgewässer-Biologen das Thema wieder aufgreifen und über den ökologischen Kenntnisstand berichten.

Walter Giger

Joint Meeting of SETAC-EUROPE

Aquatic Ecosystem Health and Management Society

Universität Potsdam/Technische Universität Berlin June 21-24, 1992

The general theme of the conference will be: Ecology, Environmental Fate, Toxicology, Bioremediation. The subject areas should focus on: Ecology and Ecotoxicology (sediment/soil testing, in situ monitoring, ecological effects, community level); Chemical Fate (experimental, modelling, interpretation); Site Resforation (bio-remediation as cost effective-technique, abandoned waste sites, hazard assessment, monitoring as cost effective-technique).

The conference will provide a forum of discussions between scientists, policy makers, regulators, and representatives from the commercial and industrial sectors.

- For further information contact:

Prof. Dr. P.-D. Hansen, Technische Universität Berlin, FB 14 Institut für Ökologie, Goerzallee 253, D-1000 Berlin 37, Telephone: 00493081696981 , Telefax: 0049308239667 